\title{
Vascular Endothelial Growth Factor-D (VEGF-D) Overexpression and Lymphatic Expansion in Murine Adipose Tissue Improves Metabolism in Obesity
}

Adri Chakraborty, ${ }^{*}$ Sheridan Barajas, ${ }^{*}$ Gabriela M. Lammoglia, ${ }^{*}$ Andrea J. Reyna, ${ }^{*}$ Thomas S. Morley, ${ }^{\dagger}$ Joshua A. Johnson, Philipp E. Scherer, ${ }^{\dagger}$ and Joseph M. Rutkowski*

From the Division of Lymphatic Biology, * Department of Medical Physiology, Texas A\&M College of Medicine, College Station; and the Touchstone Diabetes Center, ${ }^{\dagger}$ Department of Internal Medicine, University of Texas Southwestern Medical Center, Dallas, Texas

Accepted for publication

December 11, 2018.

Address correspondence to Joseph M. Rutkowski, Ph.D., Division of Lymphatic Biology, Department of Medical Physiology, Texas A\&M University College of Medicine, 345 Reynolds Bldg., MS 1114, College Station, TX 77843-1114. Email: rutkowski@tamu.edu.

\begin{abstract}
Obese adipose tissue expansion is an inflammatory process that results in dysregulated lipolysis, increased circulating lipids, ectopic lipid deposition, and systemic insulin resistance. Lymphatic vessels provide a route of fluid, macromolecule, and immune cell clearance, and lymphangiogenesis increases this capability. Indeed, inflammation-associated lymphangiogenesis is critical in resolving acute and chronic inflammation, but it is largely absent in obese adipose tissue. Enhancing adipose tissue lymphangiogenesis could, therefore, improve metabolism in obesity. To test this hypothesis, transgenic mice with doxycycline-inducible expression of murine vascular endothelial growth factor (VEGF)-D under a tightly controlled Tet-On promoter were crossed with adipocyte-specific adiponectin -reverse tetracycline-dependent transactivator mice (Adipo-VD) to stimulate adipose tissue -specific lymphangiogenesis during 16-week high-fat diet-induced obesity. Adipose VEGF-D overexpression induced de novo lymphangiogenesis in murine adipose tissue, and obese Adipo-VD mice exhibited enhanced glucose clearance, lower insulin levels, and reduced liver triglycerides. On $\beta-3$ adrenergic stimulation, Adipo-VD mice exhibited more rapid and increased glycerol flux from adipose tissue, suggesting that the lymphatics are a potential route of glycerol clearance. Resident macrophage crown-like structures were scarce and total $\mathrm{F} 4 / 80^{+}$macrophages were reduced in obese AdipoVD s.c. adipose tissue with evidence of increased immune trafficking from the tissue. Augmenting VEGF-D signaling and lymphangiogenesis specifically in adipose tissue, therefore, reduces obesityassociated immune accumulation and improves metabolic responsiveness. (Am J Pathol 2019, 189: 924-939; https://doi.org/10.1016/j.ajpath.2018.12.008)
\end{abstract}

Obesity is an epidemic-level health problem because of the comorbidities of the metabolic syndrome caused by dysfunctional expanded adipose tissue. Systemic insulin resistance and dyslipidemia are rooted in the inability of obese adipose tissue to safely store excess calories. ${ }^{1}$ Obese adipose tissue exhibits symptoms of chronic inflammation, including hypoxia, immune cell accumulation, and fibrosis, leading to the spilling over of proinflammatory cytokines and excess lipids to the system. ${ }^{2-4}$ In preclinical models, targeting adipose blood vasculature, matrix composition, or infiltrating immune cells has each proven effective in improving local adipose tissue responsiveness and, in doing so, preserving healthy systemic metabolism during obesity. ${ }^{2}$
The lymphatic vasculature is essential for maintaining tissue homeostasis through the uptake and transport of peripheral fluid, macromolecules, and immune cells. ${ }^{5}$ The presence of lymphatic endothelial cells (LECs) - themselves capable of antigen uptake and presentation-within a tissue also provides a potential platform for immune cell

\footnotetext{
Supported by the American Heart Association grant 17GRNT33671220 (J.M.R.), the Texas A\&M University Health Science Center College of Medicine and Department of Medical Physiology (J.M.R.), NIH grants R01-DK55758 (P.E.S.), R01-DK099110 (P.E.S.), and P01-DK088761-01 (P.E.S.), and the Lipedema Foundation (J.M.R., P.E.S., and A.C.).

Disclosures: None declared.
} 
communication, differentiation, and regulation. ${ }^{6}$ These roles make lymphatic vessels and LECs, through their transport and immunologic functions, critical regulators of tissue inflammation. ${ }^{7}$ Inflammation-associated lymphangiogenesis (IAL) is common in pathologies of acute and chronic inflammation. Studies in which IAL is blocked have predominantly demonstrated that it is necessary for the removal of excess fluid and cytokines and the trafficking of infiltrating immune cells from the tissue. ${ }^{7}$ Deficient lymphatic architecture and reduced lymphatic function have also been reported to cause and/or result from adipose expansion and obesity. ${ }^{8-13}$ Expansion of lymphatic vasculature or preserving lymphatic function in adipose tissue may thus provide a mechanism to regulate obesity-induced adipose inflammation and restore systemic metabolic fitness.

Lymphangiogenesis, much like blood angiogenesis, is regulated and counterregulated by multiple signaling pathways and influenced by several cells types. ${ }^{14}$ The predominant signaling mechanism, the most studied and most manipulated, is that of vascular endothelial growth factor receptor (VEGFR)3 activation by its ligands VEGF-C and VEGF-D. Blockade of VEGFR-3 and supplementation with VEGF-C/D have been used as strategies to manipulate IAL and demonstrate its importance. ${ }^{7,14}$ These studies have generally confirmed that IAL supports the resolution of inflammation, although efficacy, mechanism, and response vary from tissue to tissue. ${ }^{7}$

We have recently characterized a novel mouse model in which murine VEGF-D can be specifically induced in a tissue-specific manner through a doxycycline-controllable transgene. ${ }^{15}$ Murine VEGF-D is unique in that, unlike human VEGF-D or either species' VEGF-C, it only activates VEGFR-3 and thus has minimal ability to influence blood angiogenesis or blood vessel permeability. ${ }^{15,16}$ We have demonstrated that chronic adipose-specific VEGF-D expression leads to the development of a de novo lymphatic vasculature in multiple adipose tissues (which are mostly devoid of an endogenous lymphatic vasculature) over a period of months. ${ }^{15}$ Herein, we use this inducible adipose VEGF-D-overexpressing (Adipo-VD) mouse coupled with chronic high-fat diet feeding to identify the impacts of increased VEGFR-3 signaling and lymphatic density in obese fat. Despite equivalent weight gain, Adipo-VD mice demonstrate reduced systemic insulin resistance, liver lipid deposition, and adipose immune accumulation compared with their littermates. These findings highlight the importance of lymphatic expansion in maintaining tissue homeostasis and identify a new potential target in the amelioration of the metabolic syndrome associated with obesity.

\section{Materials and Methods}

\section{Animals}

Tetracycline-response element (TRE) promoter VEGF-D mice (TRE-VEGF-D) were generated as described. ${ }^{15}$ TRE-VEGF$\mathrm{D}$ mice were mated with adiponectin-promoter (AdipoQ) advanced reverse tetracycline-dependent transactivator (rtTA) mice for adipocyte-specific expression. ${ }^{17,18}$ Mice were continuously backcrossed to $\mathrm{C} 57 \mathrm{~B} 1 / 6 \mathrm{~J}$ for a minimum six generations, with typically male AdipoQ-rtTA ${ }^{+/}$, TRE-VEGF-D ${ }^{+/+}$mice breeding with wild-type females.

All mice used were hemizygous for the TRE-VEGF-D transgene, but they were either wild type or hemizygous for the AdipoQ-rtTA transgene. All experiments were performed with males or females (as indicated) and their immediate littermates (or combined litters at time of weaning), typically in groups of two age-matched cages (thus, four versus six or five versus five groups). Mice were housed in Association for Assessment and Accreditation of Laboratory Animal Care International-approved housing with 12-hour light-dark cycles and ad libitum access to water and food. Doxycycline-containing diets were supplied to all mice in all studies so as to normalize any effects of doxycycline itself on metabolism. Custom chow diet (Bio-Serv F4107; Bio-Serv, Flemington, NJ) or $60 \% \mathrm{kcal}$ from fat [D16042102, lard based (supplemented D12492) used throughout or modified with $100 \%$ of lard oil replaced with $100 \%$ palm oil for high saturated fat studies (D16012108) or 66\% safflower oil for unsaturated-enriched studies (D16042304); Research Diets, Inc., New Brunswick, NJ] containing $600 \mathrm{mg} / \mathrm{kg}$ doxycycline was supplied to all mice for the time periods described. Diets were initiated at 8 to 12 weeks of age, with most studies performed after 16 weeks of diet. All animal study protocols were approved by the Institutional Animal Care and Use Committee at Texas A\&M University (College Station, TX) or the University of Texas Southwestern Medical Center (Dallas, TX).

\section{Body Composition Analysis and Systemic Respiration}

Body composition of mice was measured biweekly using an EchoMRI 100H (EchoMRI LLC, Houston, TX) or Bruker LF50 BCA-Analyzer (Bruker, Billerica, MA) immediately before euthanization. Composition is reported as percentage of total mass lean, fat, and fluid. Adipose depot wet weights were measured on dissection.

To measure metabolic activity, food and water intake, and locomotion, mice were housed for 72 hours (24-hour acclimatization, 48-hour reported measures) in TSE PhenoMaster cages (TSE Systems, Inc., Chesterfield, MO) with recorded ad libitum access to their respective diets and water.

\section{Tissue, Protein, and RNA Preparation}

After exsanguination under isoflurane, tissues were harvested and either immediately fixed in 10\% buffered zinc formalin or flash frozen in liquid $\mathrm{N}_{2}$ for protein and RNA extractions. The entire s.c. inguinal white adipose tissue depot-equivalent to mammary pads 4 and 5 in females - was excised and represented as IWAT throughout. The gonadal visceral white adipose tissue depot (attached to the male epididymis or female 
uterus) was excised. The interscapular brown adipose depot was trimmed of surrounding white adipose tissue and represented as BAT throughout. Proteins were extracted by homogenization in TNET buffer (Tris, $50 \mathrm{mmol} / \mathrm{L} ; \mathrm{NaCl}, 150$ mmol/L; EDTA, 1 mmol/L; Triton X-100, 1\%; pH 8.0) with protease and phosphatase inhibitors added (ThermoFisher, Waltham, MA). For adipose depots, detergent-free buffer was first used, the samples were spun to remove excess lipid, $10 \%$ Triton X-100 was added (for a final 1\%), and pellet was rehomogenized. All proteins were normalized to 3 to $5 \mathrm{mg} /$ $\mathrm{mL}$ in TNET after bicinchoninic acid assay; measures of protein concentration were read on a SpectraMAX 250 (Molecular Devices, San Jose, CA). Liver and muscle (total quadricep or total gastrocnemius, as indicated) proteins were similarly processed in full TNET buffer. RNAs were extracted using a Zymo Direct-zol RNA Miniprep Plus, according to the manufacturer's instructions (Zymo Research, Irvine, CA). Reverse transcription of $1 \mu \mathrm{g}$ RNA was performed according to the iScript cDNA Synthesis kit instructions (BioRad Laboratories, Inc., Hercules, CA).

\section{Protein Quantitation by Western Blot Analysis}

Tissue protein extracts were heated for 5 minutes at $95^{\circ} \mathrm{C}$ in $5 \times$ Laemmli buffer containing $100 \mathrm{mmol} / \mathrm{L}$ dithiothreitol. Protein $(5$ to $50 \mu \mathrm{g})$ was loaded into wells of Mini-Protean TGX, any kDa (Bio-Rad Laboratories, Inc.), or Novex WedgeWell $4 \%$ to $20 \%$ Tris-Glycine (Invitrogen, Carlsbad, CA) gels and separated by gel electrophoresis. Gels were transferred to nitrocellulose membranes (Bio-Rad Laboratories, Inc.); incubated with primary antibodies against uncoupling protein 1 , phosphorylated hormone-sensitive lipase, hormone-sensitive lipase, phosphorylated Akt, or Akt overnight (Table 1); labeled by the appropriate IR800 Dye (LI-COR Biosciences, Lincoln, NE) conjugated secondary antibodies; and scanned on an Odyssey (LI-COR Biosciences). Densitometry was measured using Image Studio software version 4.0 (LI-COR Biosciences) and normalized to the $\beta$-actin signal for each sample (indicated with an IR700 secondary).

\section{Quantitative Real-Time RT-PCR}

Biorad iTaq universal SYBR Green supermix (Bio-Rad Laboratories, Inc.) was used with adipose tissue cDNAs in 5 $\mu \mathrm{L}$ reactions on a 384-well 7900T quantitative PCR machine (Applied Biosystems, Foster City, CA). Analyses in adipose tissues used $U b c$ as the most reproducible universal control, with expression represented as $2^{-\Delta \Delta \mathrm{Ct}}$ compared with the - rtTA littermates. Primer sequences are listed in Table 2.

\section{Histology and Immunofluorescence}

After 24-hour fixation, tissues were rinsed in water and stored in 50\% ethanol until processed for paraffin embedding. Sections $(3-4 \mu \mathrm{m}$ thick) were cut from all tissues.
Table 1 Antibodies Used in This Study

\begin{tabular}{|c|c|c|c|}
\hline Antigen & Source & Catalog number & RRID \\
\hline UCP-1 & $\begin{array}{l}\text { Abcam } \\
\quad \text { (Cambridge, UK) }\end{array}$ & 10983 & AB_2241462 \\
\hline Akt & $\begin{array}{l}\text { Cell Signaling } \\
\text { (Danvers, MA) }\end{array}$ & 4685 & AB_2225340 \\
\hline pAkt & Cell Signaling & 4060 & AB_2315049 \\
\hline$\beta$-actin & Sigma & $\mathrm{A} 2228$ & $A B \_476697$ \\
\hline HSL & Cell Signaling & 4107 & AB_2296900 \\
\hline pHSL & Cell Signaling & 4139 & AB_2135495 \\
\hline CD16/CD32 & $\begin{array}{l}\text { BD Biosciences } \\
\text { (San Jose, CA) }\end{array}$ & 553141 & AB_394656 \\
\hline CD45.1 & BD Biosciences & 562452 & AB_11152958 \\
\hline CD45.2 & BD Biosciences & 561874 & AB_10894189 \\
\hline $\mathrm{F} 4 / 80$ & BD Biosciences & 565410 & $A B \_2687527$ \\
\hline CD19 & BD Biosciences & 561737 & $A B \_10896279$ \\
\hline CD3e & BD Biosciences & 561100 & $A B \_10562036$ \\
\hline CD11c & BD Biosciences & 560584 & $A B \_1727422$ \\
\hline LYVE-1 & $\begin{array}{l}\text { R\&D Systems } \\
\text { (Minneapolis, MN) }\end{array}$ & AF2125 & AB_2297188 \\
\hline Endomucin & $\begin{array}{l}\text { Santa Cruz } \\
\text { Biotechnology } \\
\text { (Dallas, TX) }\end{array}$ & sc- 65495 & AB_2100037 \\
\hline Podoplanin & R\&D Systems & AF3244 & AB_2268062 \\
\hline VEGFR-3 & R\&D Systems & AF743 & AB_355563 \\
\hline Prox-1 & $\begin{array}{l}\text { AngioBio } \\
\quad \text { (San Diego, CA) }\end{array}$ & $11-002 P$ & AB_10013720 \\
\hline Mac-2 & $\begin{array}{l}\text { Cedarlane } \\
\quad \text { (Burlington, NC) }\end{array}$ & CL8942AP & AB_10060357 \\
\hline
\end{tabular}

HSL, hormone-sensitive lipase; LYVE-1, lymphatic vessel endothelial hyaluronan receptor 1; Mac-2; pAkt, phosphorylated Akt; pHSL, phosphorylated HSL; Prox-1, prospero homeobox protein 1; RRID, research resource identifier; UCP-1, uncoupling protein 1; VEGFR-3, vascular endothelial growth factor receptor-3.

Adipose depots were sectioned to maximize area (and include the lymph node, in the case of inguinal adipose).

Tissue sections were stained by Masson's trichrome method for visualizing adipose tissue and matrix density. For immunofluorescence, sections were deparaffinized, rehydrated, and subjected to 10 minutes of citrate buffer boiling (Vector Labs, Burlington, CA) antigen retrieval when necessary. Tissues were labeled with primary antibodies against LYVE-1, endomucin, podoplanin, VEGFR3, Prox-1, and Mac-2 (Table 1), with fluorophoreconjugated secondary detection. Immunofluorescence was imaged using an AxioObserver fluorescence microscopy system and MRc camera (Zeiss, Thornwood, NY) or an Eclipse E600 microscope (Nikon, Melville, NY), and images were captured using cellSens Standard version 1.18 imaging software (Olympus, Waltham, MS).

Whole-mount preparations of adipose tissue were first fixed for 24 hours, then maintained in $0.3 \%$ Triton X-100 in phosphate-buffered saline for 24 hours before the tissues were cut into $<1-\mathrm{cm}^{3}$ size and incubated in antibodies and against LYVE-1 for 4 days; this was followed by rinsing and 48-hour secondary antibody incubation. Adipose pieces 
Table 2 Primer Sequences Used for Quantitative RT-PCR Analysis of Murine Adipose Tissues

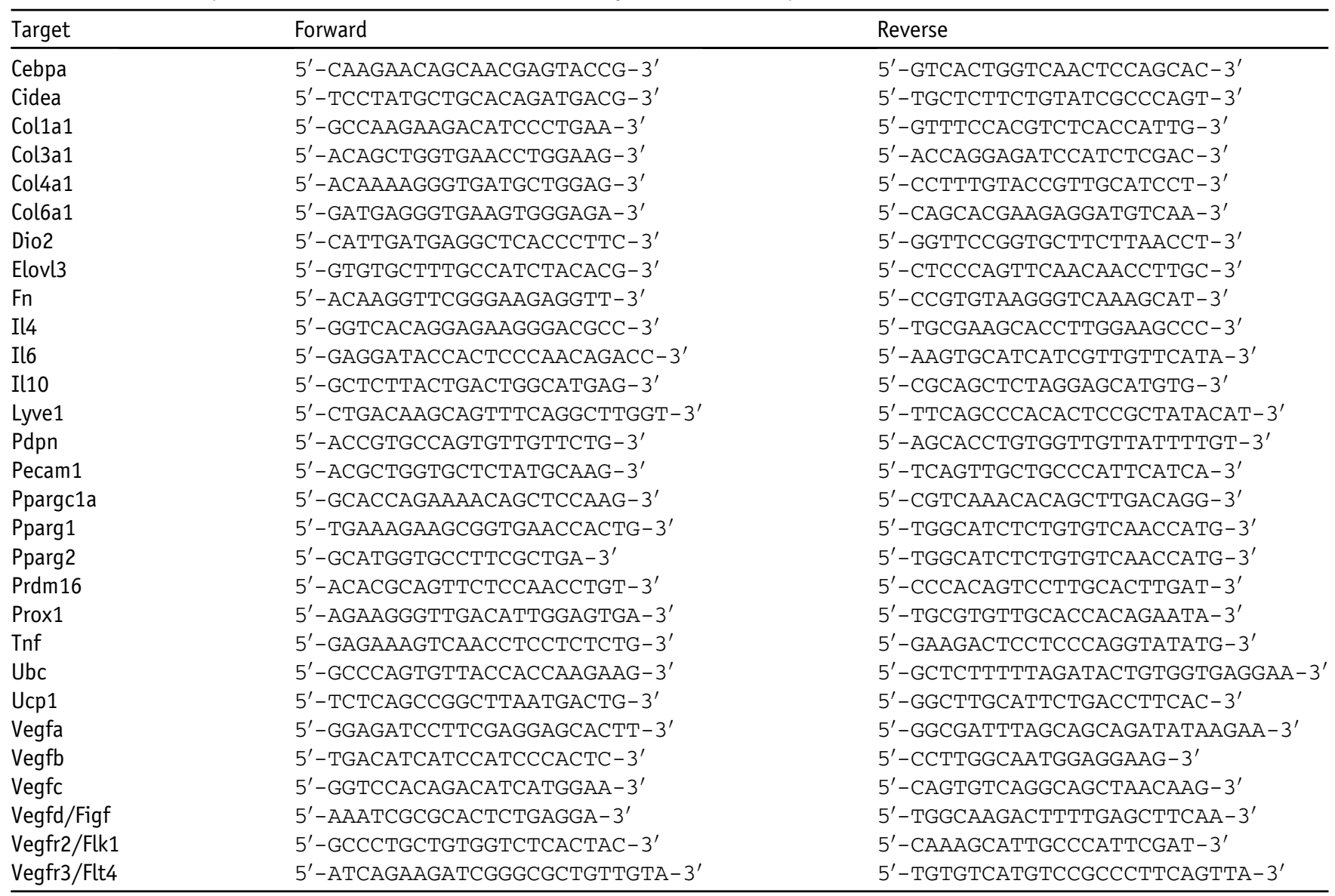

Common names and variant names provided. All sequences were verified through National Center for Biotechnology Information Primer-BLAST ( $h$ ttps://www. ncbi.nlm.nih.gov/tools/primer-blast), and single products were confirmed with a dissociation step after amplification.

were imaged on a Zeiss Stallion Digital Imaging Workstation using a CoolSnap HQ Camera (Photometrics, Tuscon, AZ) with external ventilation.

\section{Oral Glucose, Lipid Clearance, and Insulin Tolerance Tests}

An oral load of $1.4 \mathrm{~mol} / \mathrm{L}$ glucose solution at $10 \mu \mathrm{L} / \mathrm{g}$ or Intralipid (Baxter, Deerfield, IL) at $10 \mu \mathrm{L} / \mathrm{g}$ was given after a 3-hour fast. For insulin, an i.p. injection of $0.75 \mathrm{U} / \mathrm{kg}$ was delivered. Lipid experiments were performed 2 weeks before and glucose experiments were performed 1 week before the terminal end point. Blood samples were collected from the tail tip at the times indicated in the experiments. Glucose and serum triglycerides were measured by Sigma Diagnostics Glucose reagent and Infinity reagent (ThermoFisher) spectrophotometric assay, respectively. Serum insulin concentrations were determined by enzyme-linked immunosorbent assay, according to the manufacturer's instructions (Crystal Chem, Downer's Grove, IL). Serum cholesterol levels were measured by Amplex Red Cholesterol Assay Kit (ThermoFisher).

\section{Tissue Lipid Uptake and 0xidation}

To track the uptake and oxidation of triglyceride from circulation, ${ }^{3} \mathrm{H}$-triolein (PerkinElmer, Waltham, MA) was added to Intralipid for i.v. infusion $(2 \mu \mathrm{Ci}$ per mouse in 200 $\mu \mathrm{L}$ of $5 \%$ Intralipid) after a 5 -hour fast, as previously described. ${ }^{19}$ Blood was collected at $1,2,5,10$, and 15 minutes after infusion to analyze systemic clearance. Mice were immediately euthanized by exsanguination under isoflurane, and tissues samples were collected and weighed. Tissues were homogenized in 2:1 chloroform/methanol, mixed with $1 \mathrm{~mol} / \mathrm{L} \mathrm{CaCl}_{2}$, and centrifuged. The lipid and aqueous fractions were thus separated, representing absorbed and oxidized triglycerides, respectively; measured for tritium decays per minute on a PerkinElmer Tri-Carb 3110 TR liquid scintillation counter; and normalized to tissue mass.

\section{Lipolysis Induction}

To stimulate lipolysis in vivo, mice were administered a $\beta-3$ adrenergic receptor agonist, $1 \mathrm{mg} / \mathrm{kg}$ CL-316,243 (Sigma, St. Louis, MO), intraperitoneally after a 5-hour fast. Blood 
samples were collected at $0,15,30,45,60$, and 120 minutes after injection and measured for glucose (as above), glycerol by free glycerol assay (Sigma), and nonesterified fatty acids (Wako Diagnostics, Mountain View, CA).

Adipose depots were tested for lipolysis ex vivo using $\beta-1$ adrenergic stimulation with isoproterenol $(10 \mu \mathrm{mol} / \mathrm{L})$. Depots were excised, weighed, placed into $37^{\circ} \mathrm{C}$ basal Dulbecco's modified Eagle's medium, and grossly minced. After 1 hour, $10 \%$ of the media was removed and replaced with media containing isoproterenol. After 1 hour, that media, and the 1-hour media, were measured for glycerol by free glycerol assay (Sigma). Results are expressed as fold change compared with unstimulated samples.

The stromal vascular fraction of Adipo-VD and littermate inguinal adipose tissue was isolated, cultured, and differentiated to white- and brown-like adipocytes, as previously described. ${ }^{20,21}$ White cell differentiation was induced using a cocktail of insulin, dexamethasone, isobutylmethyxanthine, and rosiglitazone (all from Sigma) and maintained with 5 $\mu \mathrm{g} / \mathrm{mL}$ insulin. Brown differentiation was induced with a cocktail of dexamethasone, isobutylmethyxanthine, and indomethacin. Brown cells were maintained with added insulin and triiodothyronine. Doxycycline $(1 \mu \mathrm{mol} / \mathrm{L}$ final $)$ was provided in the media throughout differentiation. Ten days after initiating differentiation, lipolysis was stimulated by isoproterenol. Glycerol secretion was measured as with the ex vivo tissues, above.

\section{Lymph Node Fluorescence Uptake}

Intradermal injections $(20 \mu \mathrm{L})$ of $2 \mathrm{mg} / \mathrm{mL}$ fluorescein isothiocyanate dextran $(70 \mathrm{kDa}$; Molecular Probes, Eugene, OR) were made subcutaneously into the top of the right hind foot. Mice were sacrificed 30 minutes later (as above), and the popliteal, inguinal, and axillary lymph nodes were collected from both sides of the mouse. Nodes were homogenized in radioimmunoprecipitation assay buffer, and the fluorescence density at $488 \mathrm{~nm}$ was measured on a SpectraMAX Gemini fluorescence plate reader (Molecular Devices, Sunnyvale, CA) with the negative side subtracted as baseline. Protein concentrations of the same sample were measured by bicinchoninic acid assay and used to normalize fluorescence.

\section{Flow Cytometry}

Tissues were collected, minced, and incubated for 1 hour in digestion buffer $(100 \mathrm{mmol} / \mathrm{L}$ HEPES, $120 \mathrm{mmol} / \mathrm{L} \mathrm{NaCl}, 50$ $\mathrm{mmol} / \mathrm{L} \mathrm{KCl}, 5 \mathrm{mmol} / \mathrm{L}$ glucose, $1 \mathrm{mmol} / \mathrm{L} \mathrm{CaCl} 2,1.5 \%$ bovine serum albumin, and $1 \mathrm{mg} / \mathrm{mL}$ Collagenase D; Sigma) with regular gentle agitation at $37^{\circ} \mathrm{C}$. Digests were strained through $40-\mu \mathrm{m}$ cell strainers, red blood cells were lysed (in $155 \mathrm{mmol} / \mathrm{L} \mathrm{NH}_{4} \mathrm{Cl}, 10 \mathrm{mmol} / \mathrm{L} \mathrm{KHCO}_{3}$, and $0.1 \mathrm{mmol} / \mathrm{L}$ EDTA), and cell pellets were resuspended in flow buffer (phosphate-buffered saline with $0.5 \%$ fetal bovine serum and $0.1 \mathrm{mmol} / \mathrm{L}$ EDTA). All samples were first blocked using
anti-CD16/CD32 for 10 minutes. Cells were then incubated with antibodies against CD45.1, CD45.2, F4/80, CD19, CD3e, and CD11c, with dead cells stained with DAPI (Table 1). Cell preparations were analyzed on a BD LSR Fortessa X-20 flow cytometer (BD Biosciences, San Jose, CA). In immune population analysis experiments, 10,000 immune events were analyzed. In CD45.1 infusion experiments, 3 million splenocytes sterilely isolated from CD45. $1^{+}$ FVB mice (prepared as cells above) were injected intravenously 24 hours before euthanasia. In these experiments, the entire volume of adipose sample was analyzed for total cell numbers of the respective populations. Analysis was performed by first gating for immune populations by FS/SS, singlets, and living cells that were CD45.2 or CD $45.1^{+}$before subpopulation identification as a fraction of total $\left(\right.$ or $\left.\mathrm{CD} 45^{+}\right)$ events.

\section{Image Analysis}

Image analysis was performed using ImageJ software version $1.52(\mathrm{NIH}$, Bethesda, MD; http://imagej.nih.gov/ij) to determine the positive immunolabeled areas for LYVE1, endomucin, or Mac-2 on sections from adipose depots, muscle, heart, liver, and pancreas. For each quantified tissue, four to five random fields were captured at $\times 10$ magnification (as above) as the tissue area permitted at the same exposure settings for each fluorophore. Larger vessels and tissue defects were purposefully avoided. In ImageJ, positive labeling was identified by a fluorescence threshold and measured as that area/total area. Adipocyte size was calculated using the Adiposoft plug-in for ImageJ on equivalent random imaging captured from trichrome-labeled s.c. adipose sections. Images were first averaged per animal, then per group.

\section{Statistical Analysis and Data Presentation}

In all studies, all mice carry equal copies of the TRE-VEGF$\mathrm{D}$ transgene (VD). The lack or the presence of the AdipoQrtTA transgene designates Adipo-VD versus littermates, respectively. All studies tried to use minimally four versus six or five versus five groups of mice [except metabolic cage studies that were two versus three (one wild-type mouse refused to eat)]. Mice were genotyped for the rtTA transgene at termination, which altered some planned group numbers. In each experiment, statistical significance was tested by unpaired $t$-test comparisons, assuming unequal variance between the Adipo- $\mathrm{VD}^{+}$and littermate groups using GraphPad Prism software version 7.05 (GraphPad Software, La Jolla, CA). For grouped column data, multiple comparison $t$-tests were performed with the recommended Holm-Sidak correction in GraphPad Prism software. For time-dependent data or grouped data, analysis of variance was applied with Bonferroni post hoc analyses to determine the significance. Data are presented as means $\pm \mathrm{SD}$; timedependent graphs display SEMs only for visual clarity. 
Average areas under the curves were calculated using the trapezoidal method and provided when significant. $P<0.05$ was considered significant.

\section{Results}

\section{VEGF-D Overexpression by Adipocytes Drives Marked Adipose Tissue Lymphatic Expansion in Obesity}

Obesity-associated inflammation increases gene expression of the VEGFR-3 ligands, VEGF-C and VEGF-D (Figure 1A), but elicits no discernible lymphatic expansion in male murine s.c. adipose tissue. ${ }^{2,23}$ VEGF-D overexpression can induce lymphatic expansion in adipose tissue, but only after a period of 2 to 4 months in AdipoVD mice. ${ }^{15}$ To test the impact of increased lymphatics on adipose function and inflammation in obesity, Adipo-VD mice were placed on a $60 \% \mathrm{kcal}$ from fat diet containing $600 \mathrm{mg} / \mathrm{kg}$ doxycycline for 4 months. VEGF-D expression was significantly increased in white s.c. inguinal (Figure 1B), visceral gonadal (Supplemental Figure S1A), and interscapular BAT depots (Figure 1C). This resulted in increased expression of RNA for lymphatic endothelial cell genes Lyve1, Proxl, and Vegfr3, which significantly increased in these depots, with BAT exhibiting the highest fold change. Immunofluorescence confirmed a marked increase in lymphatic structures in s.c. inguinal adipose (Figure 1D), ranging from full infiltration to discontinuous groups of LECs (Supplemental Figure S1B). Image analysis for LYVE- $1^{+}$areas demonstrated a significant increase in lymphatics and no change in blood capillary density, measured by endomucin ${ }^{+}$area (Figure 1E). In brown adipose, lymphatic expansion was tremendous, with the entire tissue consistently filling with LECs (Figure 1F). Quantitation of LYVE- $1^{+}$area confirmed a significant increase in these structures, with no significant impact on the brown adipose endomucin-labeled blood capillary network (Figure 1G). Whole-mount imaging identified LYVE- $1^{+}$cells in inguinal adipose tissue, previously described as macrophages, ${ }^{24}$ throughout the tissue of wild-type littermates (Figure 1H). LYVE-1 ${ }^{+}$lymphatic structures, ranging from capillary-like structures to lymphatic sheets and often demonstrating the common lymphatic hexagonal patterning, were identified in IWAT (Figure $1 \mathrm{H}$ and Supplemental Figure S1B). The dense lymphatic patterning in BAT cross-sections appeared as sheets when imaged by whole-mount imaging (Supplemental Figure S1C). Despite some increases in lymphatic gene expression, no LYVE- ${ }^{+}$structures were identified by immunofluorescence in male gonadal adipose depots (data not shown). New lymphatic structures in adipose were confirmed to be positive for other LEC markers, podoplanin and Prox-1 (Figure 1I) and VEGFR-3 (Figure $1 \mathbf{J}$ ). Changes in lymphatic density were limited to adipose tissue depots, with no differences in LYVE-1 ${ }^{+}$ area or intensity measured in the liver sinusoids
(Supplemental Figure S2, A and B), heart (Supplemental Figure S2C), intestine (Supplemental Figure S2D), skeletal muscle, or pancreas (data not shown). Lymphatic collecting vessel transport was not inhibited by elevated VEGF-D, as measured by long-distance transport of 70$\mathrm{kDa}$ dextran from the periphery (Supplemental Figure S2E). The adipose specificity, elevation of VEGF-D levels, and degree of lymphangiogenesis were similar to previous characterization under chow-fed conditions, ${ }^{15}$ suggesting that concurrent high-fat feeding itself does not greatly inhibit lymphatic expansion.

\section{Adipo-VD Mice Exhibit Improved Glucose Disposal and Insulin Sensitivity in Obesity}

Chronic high-fat diet feeding is used in mice to drive obesity and the metabolic syndrome. Adipo-VD mice gained equivalent weight to their control littermates over 4 months of high-fat diet (Figure 2A). Body mass composition measurements revealed a small increase in adiposity in obese Adipo-VD mice (Figure 2B). Similar to the findings in chow (Supplemental Figure S3A), adiposity appeared to be balanced across all depots, with no single weighed depot demonstrating a notable difference in mass (Figure 2C). Adipo-VD mice exhibited no systemic edema because overall free water was reduced. The average adipocyte lipid droplet radius measured on trichrome-stained inguinal adipose tissue sections was equivalent in Adipo-VD mice (Supplemental Figure S3B).

When challenged by an oral glucose tolerance test, male Adipo-VD mice consistently demonstrated significantly improved glucose clearance (Figure 2, D and E). Insulin levels during the test were not greatly different (Figure 2, F and G). Female obese Adipo-VD mice also demonstrated improved glucose handling (Supplemental Figure S4, A and B). Improved glucose handling during loading was only seen when challenged in the obese state: chow-fed mice demonstrated no difference (Supplemental Figure S4C), and basal blood glucose levels after a 3-hour fast were equivalent in Adipo-VD mice on either chow or high-fat doxycycline diets (Supplemental Figure S4D).

Reduced insulin signaling is characteristic of the metabolic syndrome. Fed insulin levels were reduced in obese Adipo-VD mice (Figure 2H). No measured Adipo-VD tissues demonstrated an increase in Akt phosphorylation after insulin injection compared with littermates (Figure 2I). An insulin tolerance test demonstrated a small difference in glucose levels, but no cumulative difference once the areas under the curve were calculated (Figure 2, J and K). AdipoVD mice fed doxycycline-containing high-fat diet for 1 month-insufficient to increase lymphatic density but with elevated local VEGF-D expression-demonstrated no change in glucose handling (Figure 2L). Combined, longterm VEGF-D overexpression, specifically in the adipose tissue and necessary to expand lymphatics, appears necessary to improve glucose loading response in obese mice. 
A

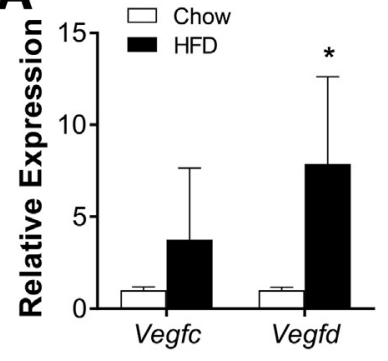

B

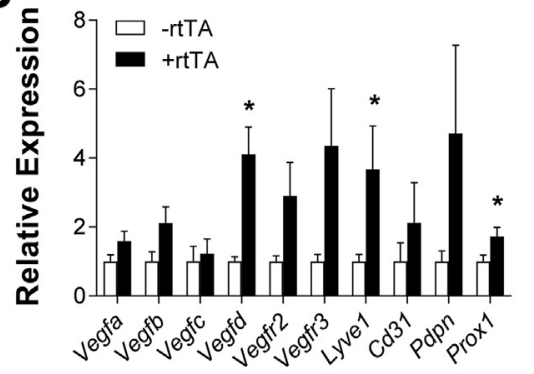

C

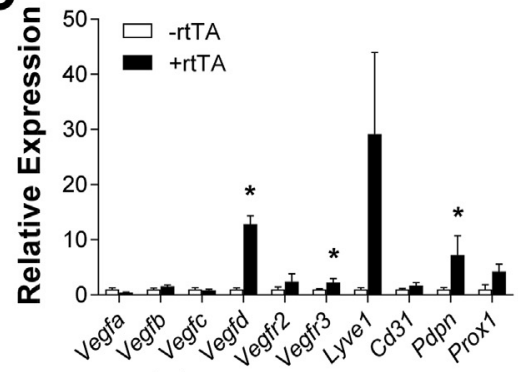

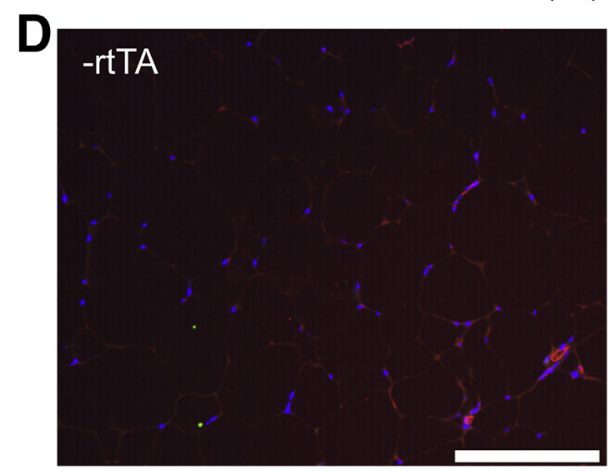

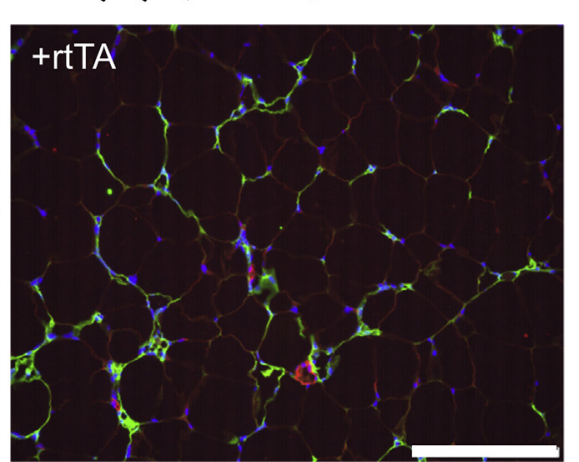

E
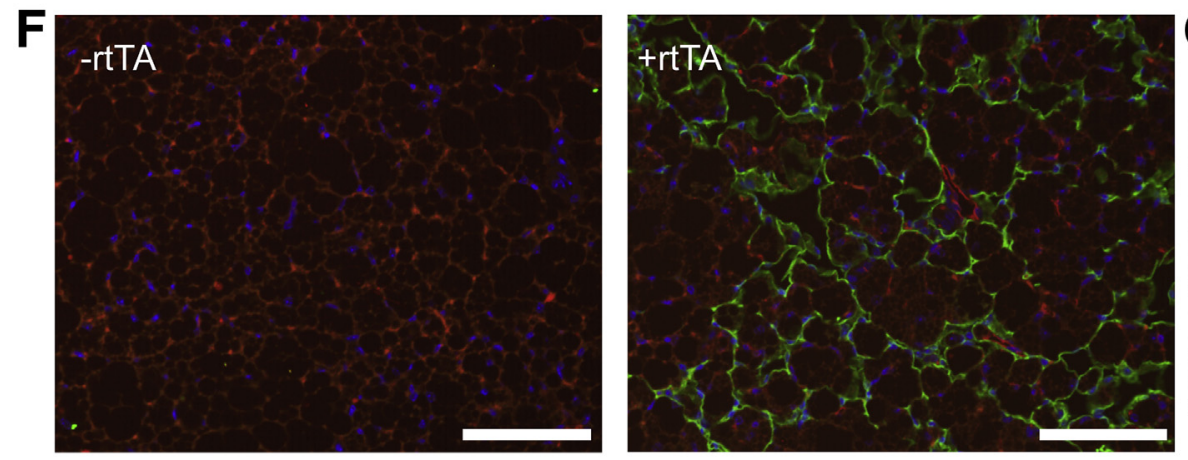

\section{G}
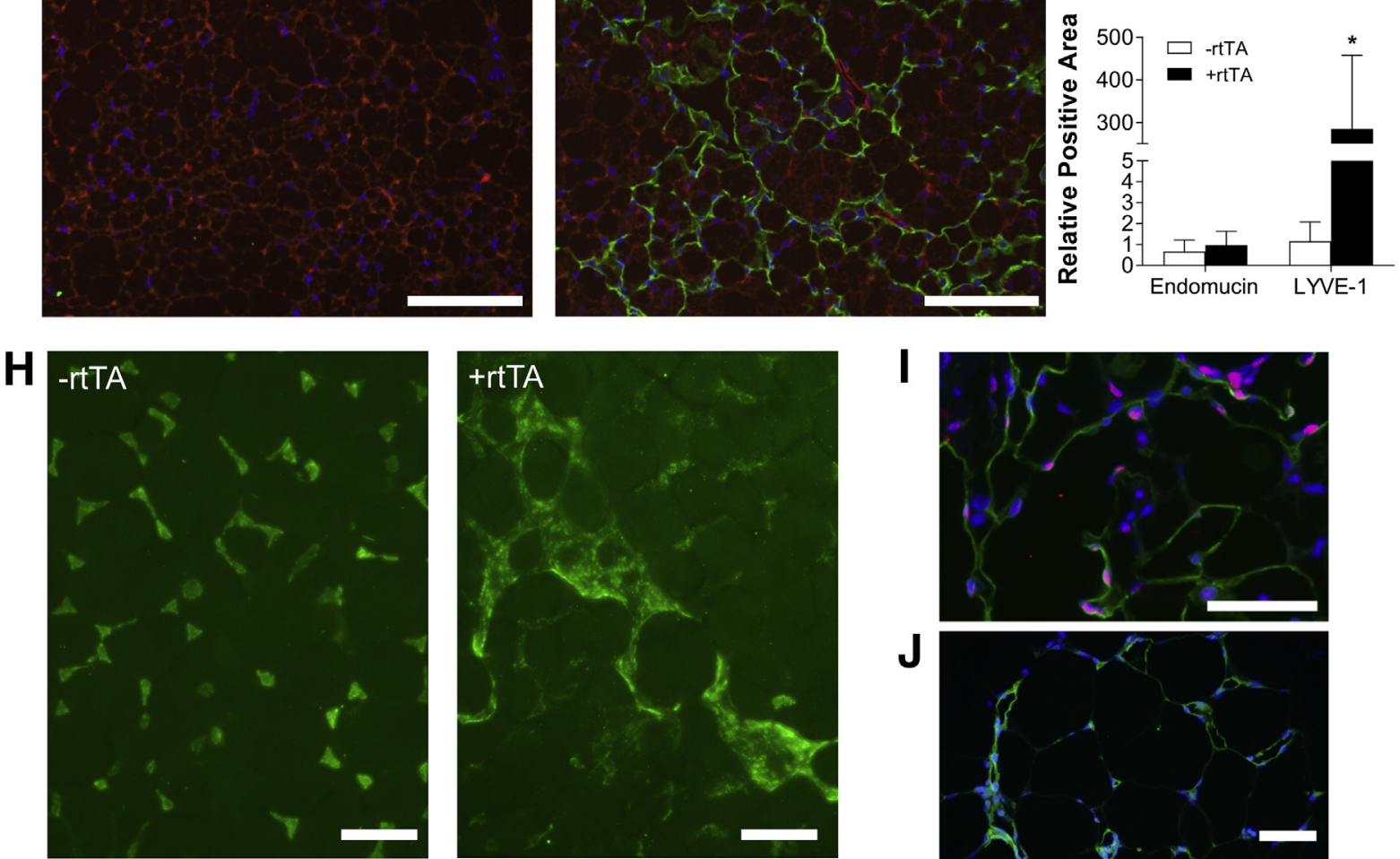

Figure 1 Inducible overexpression of VEGF-D in obesity expands adipose lymphatics. A: RNA expression for the lymphangiogenic VEGFR-3 ligands Vegf-c and Vegf-d is physiologically elevated in high-fat diet (HFD)-fed obese mouse inguinal adipose tissue compared with chow-fed mice. B: Induction of VEGF-D translation in Adipo-VD mice elevates Vegf-d and lymphatic endothelial cell marker RNAs in inguinal white adipose tissue. C: Brown adipose tissue exhibits higher VEGF-D RNA induction in Adipo-VD mice. D: Immunofluorescence of lymphatic (green; LYVE-1) and blood (red; endomucin) endothelium in inguinal white adipose tissue. E: Quantitation of LYVE-1- and endomucin-positive pixels on inguinal adipose tissue section images. F: Immunofluorescence of lymphatic (green; LYVE-1) and blood (red; endomucin) endothelium in interscapular brown adipose tissue. G: Quantitation of LYVE-1- and endomucinpositive pixels on brown adipose tissue section images. H: Podoplanin (green) and Prox-1 (red) labeling of lymphatic structures in brown adipose tissue. I: VEGFR-3 (green) labeling of lymphatic structures in white adipose tissue. Blue shows DAPI in all images. J: Whole-mount LYVE-1 immunofluorescence in inguinal white adipose tissue demonstrates LYVE-1+ $1^{+}$cells resident in adipose tissue. In Adipo-VD tissue, lymphatic structures form between and around adipocytes. $n=4$ (A); $n=8$ (B and $\mathbf{D}-\mathbf{G})$. ${ }^{*} P<0.05$ versus - rtTA. Scale bars: $100 \mu \mathrm{m}(\mathbf{D}, \mathbf{F}, \mathbf{I}$, and $\mathbf{J}) ; 50 \mu \mathrm{m}(\mathbf{H})$. 

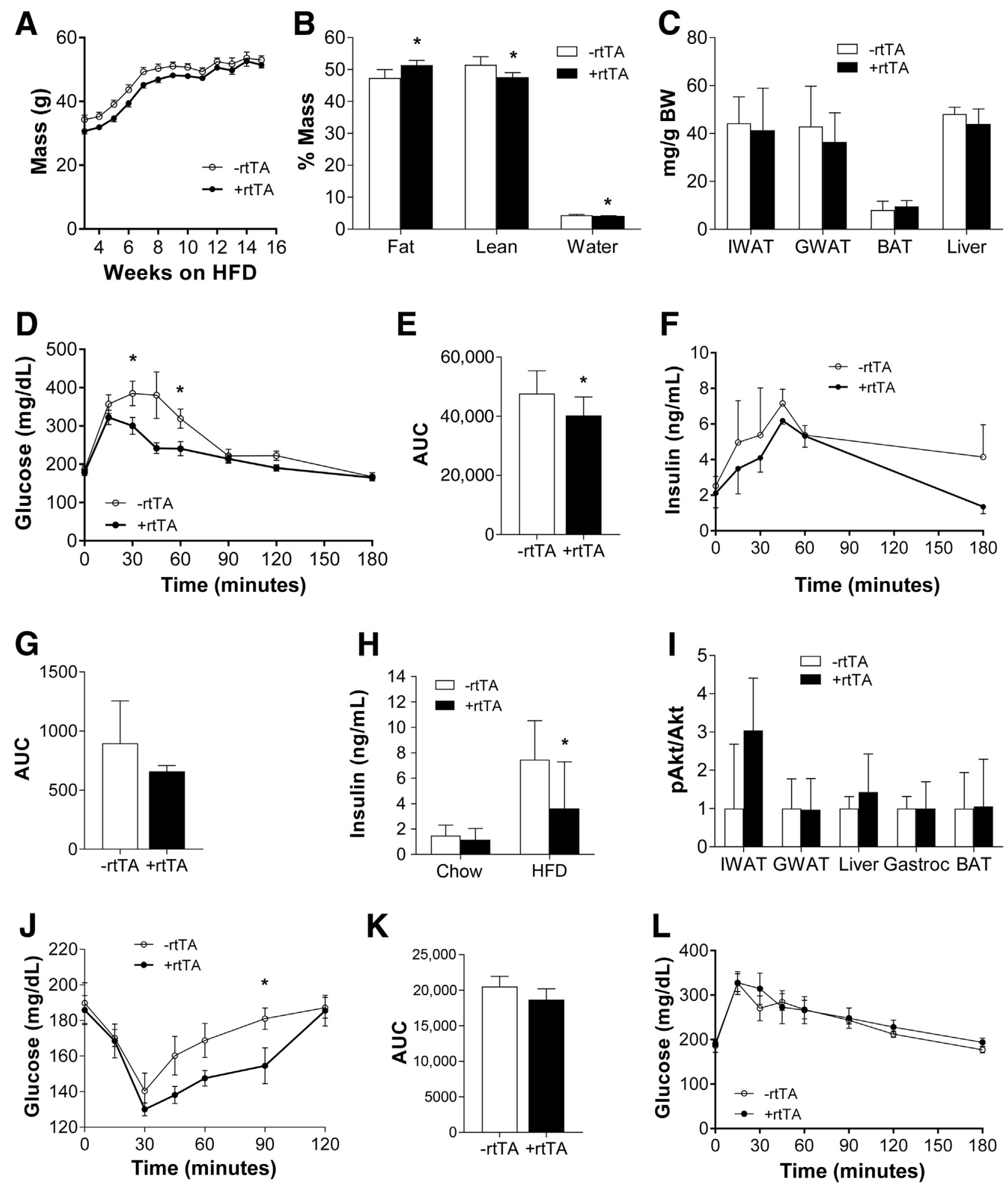

Figure 2 Adipo-VD mice exhibit equivalent weight gain, but enhanced insulin sensitivity, in obesity. A: Adipo-VD males on a high-fat diet (HFD) with doxycycline gain equivalent weight as wild-type littermates. B: Magnetic resonance imaging quantitation of body composition at 4 months of doxycycline HFD. C: Tissue weights relative to body weight (BW) at termination. D: Blood glucose levels during an oral glucose tolerance test in obese Adipo-VD mice. E: Average area under the curve (AUC) calculated from glucose levels previously tested. F: Serum insulin levels during an oral glucose tolerance test in obese Adipo-VD mice. G: Average area under the curve calculated from insulin levels during previous test. H: Fed serum insulin levels in Adipo-VD mice after 4 months of doxycycline chow or HFD. I: Phosphorylated Akt (pAkt)/total Akt ratio in obese Adipo-VD tissues [inguinal white (IWAT), gonadal white (GWAT), and brown (BAT) adipose tissues] 30 minutes after insulin injection. J: Blood glucose levels after bolus i.p. insulin injection in obese Adipo-VD mice. K: Average area under the curve from previous test. L: Oral glucose tolerance test of male Adipo-VD mice after 1-month HFD doxycycline feeding. $n=16$ (A); $n=8$ (B, C, and $\mathbf{H}) ; n=10-\mathrm{rtTA}(\mathbf{D}, \mathbf{E}$, and $\mathbf{F}) ; n=13+\mathrm{rtTA}(\mathbf{D}, \mathbf{E}$, and $\mathbf{F}) ; n=6(\mathbf{I}, \mathbf{J}$, and $\mathbf{K}) ; n=5(\mathbf{L}) .{ }^{*} P<0.05$ versus - rtTA. Gastroc, gastrocnemius muscle. 
A

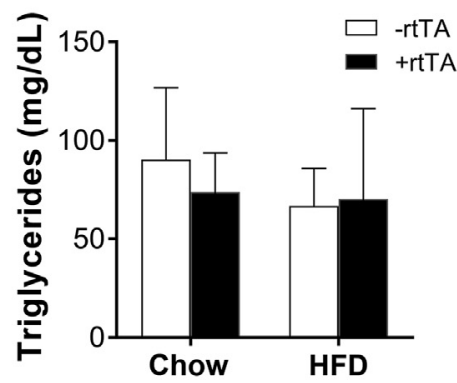

D

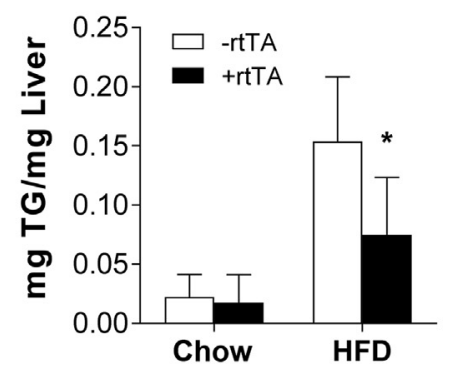

G

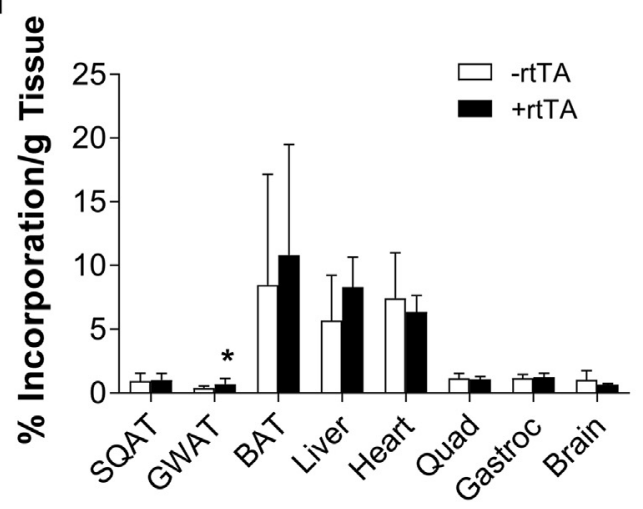

B

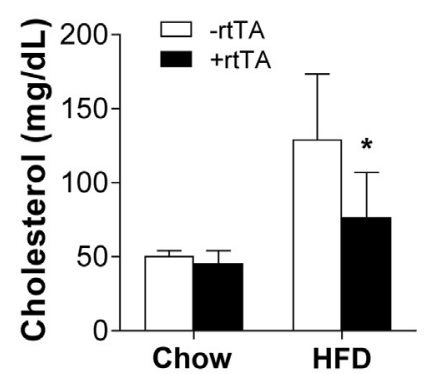

E

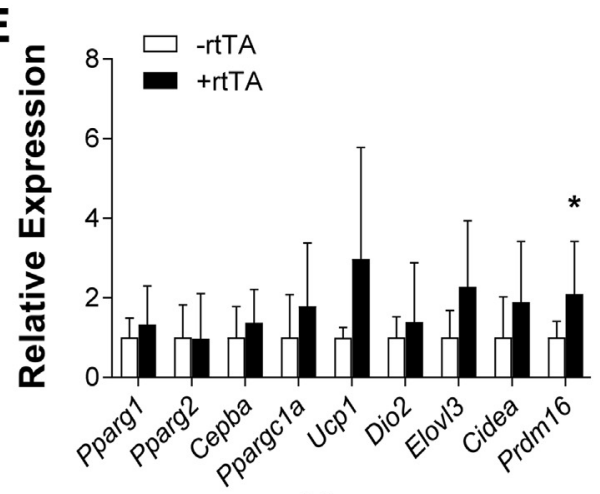

C

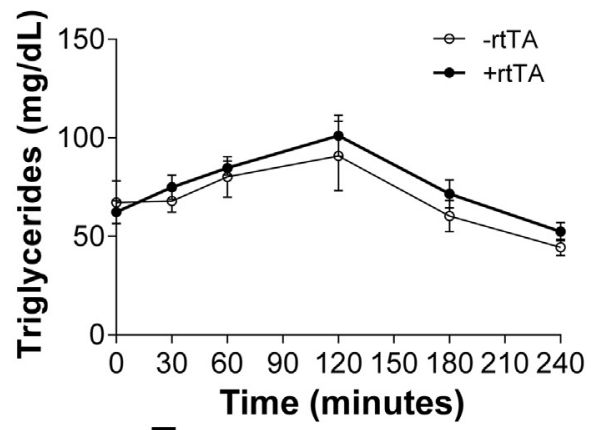

F

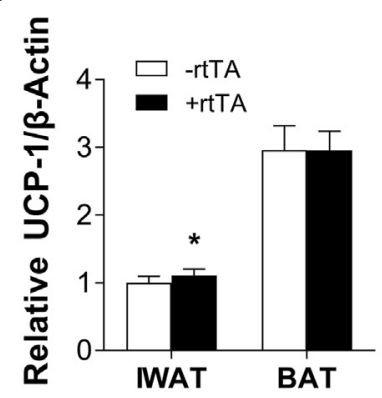

H

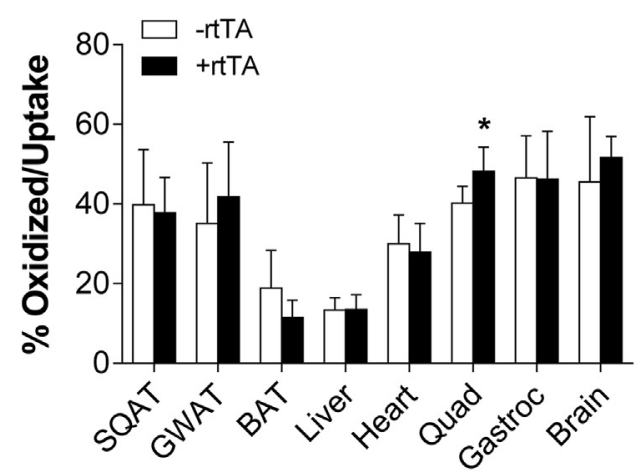

I
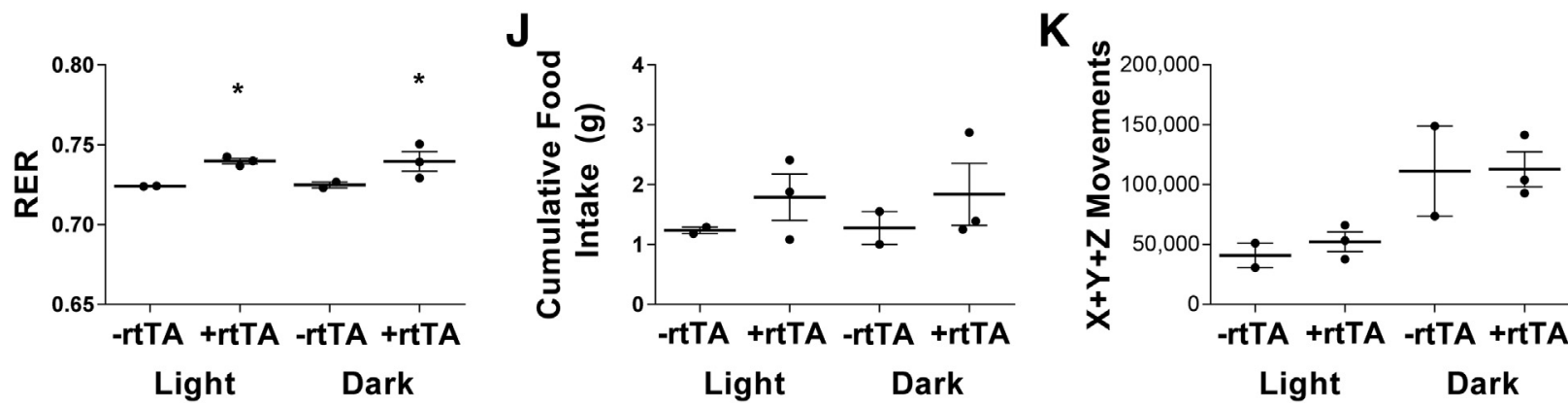

Figure 3 Lipid levels, handling, and metabolism alterations in Adipo-VD mice. A: Plasma triglyceride (TG) levels after 4 months of doxycycline chow or high-fat diet (HFD). B: Plasma cholesterol levels after 4 months of doxycycline chow or HFD. C: Plasma triglyceride levels after oral lipid loading. D: Liver triglyceride content in Adipo-VD mice after 4 months of doxycycline chow or HFD expressed per mg liver mass. E: Adipocyte determinant RNA expression in obese inguinal white adipose tissue (IWAT). F: Uncoupling protein 1 (UCP-1) protein expression in obese Adipo-VD adipose depots. G: Lipid uptake into various tissues 15 minutes after ${ }^{3} \mathrm{H}$-triolein infusion. $\mathrm{H}$ : Percentage of ${ }^{3} \mathrm{H}$-triolein incorporated into various tissue that was oxidized 15 minutes after infusion. I: Light and dark cumulative respiratory exchange ratio ( $R E R$; $\mathrm{CO}_{2}$ generation $/ \mathrm{O}_{2}$ consumption) measured in metabolic cages over 48 hours for obese littermates and Adipo-VD mice. J: Light and dark 48-hour food intake in metabolic cages. K: Light and dark cumulative movement $(\mathrm{x}+\mathrm{y})$ and rearings $(\mathrm{z})$ over 48 hours of mice in metabolic cages. $n=8-\mathrm{rtTA}(\mathbf{A}, \mathbf{B}, \mathbf{D}-\mathbf{H}) ; n=8+\mathrm{rtTA}$ $(\mathbf{A}, \mathbf{B}, \mathbf{D}-\mathbf{F}) ; n=6(\mathbf{C}) ; n=4+\mathrm{rtTA}(\mathbf{G}$ and $\mathbf{H}) ; n=2-\mathrm{rtTA}(\mathbf{I}-\mathbf{K}) ; n=3+$ rtTA $(\mathbf{I}-\mathbf{K}) .{ }^{*} P<0.05$ versus - rtTA. BAT, brown adipose tissue; Gastroc, gastrocnemius muscle; GWAT, gonadal white adipose tissue; Quad, quadriceps muscle. 

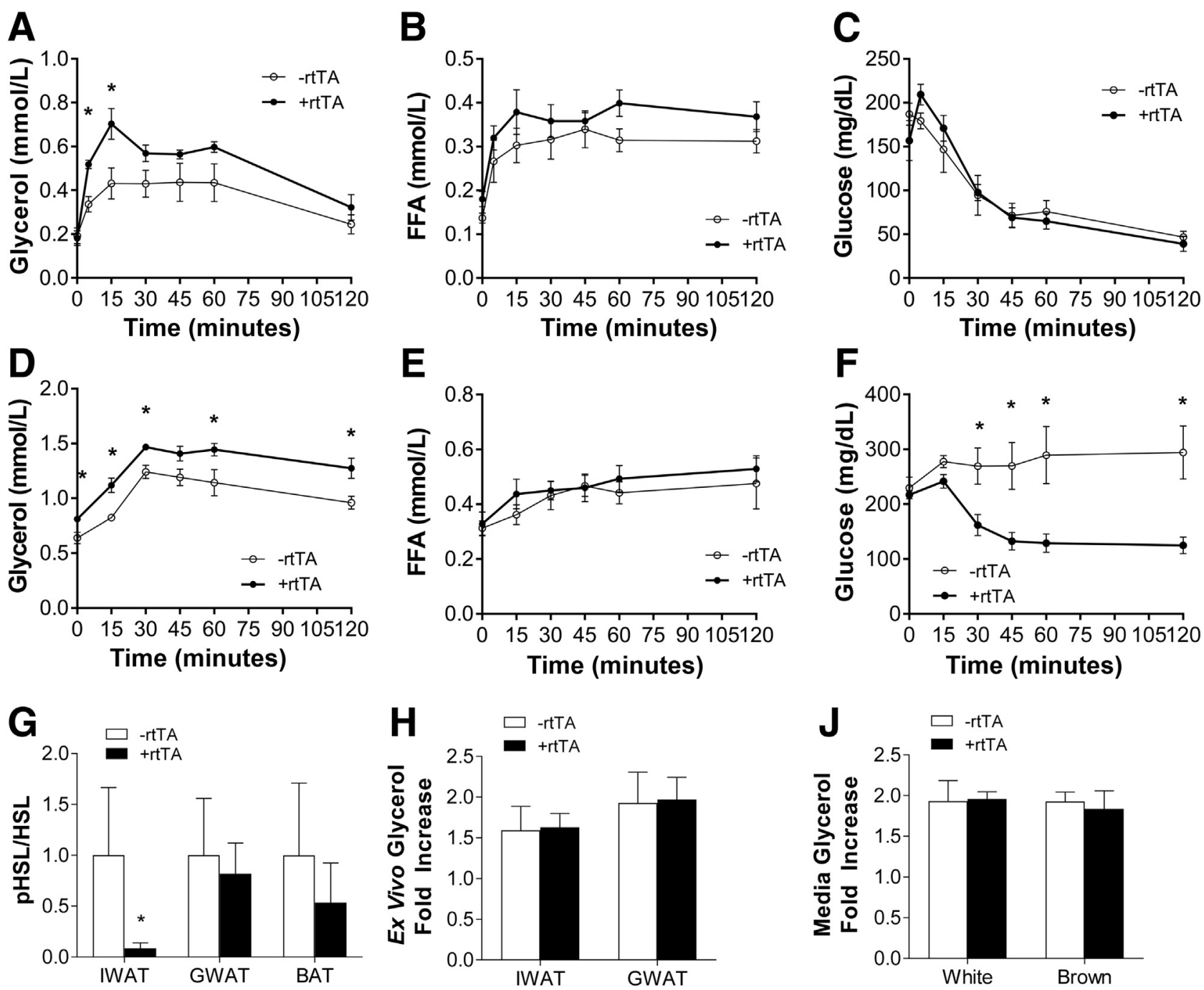

Figure 4 Alterations in adrenergic-stimulated lipolysis in Adipo-VD mice on chow and high-fat diets. A-C: Chow-fed Adipo-VD mice exhibit a more rapid and heightened glycerol appearance in blood circulation (A), but similar free fatty acid (FFA; B) and glucose (C) responses after $\beta-3$ adrenergic stimulation. D and E: Obese Adipo-VD glycerol (D) and FFA release (E) after $\beta-3$ adrenergic stimulation. F: Maintained glucose uptake during $\beta-3$ adrenergic stimulated lipolysis in obese Adipo-VD mice compared with resistant littermates. G: Phosphorylated hormone-sensitive lipase (pHSL)/total HSL in adipose depots [inguinal white (IWAT), gonadal white (GWAT), and brown (BAT) adipose tissues] of obese Adipo-VD mice 30 minutes after insulin injection. H: Fold glycerol increase in the media of isoproterenol-stimulated lipolysis in adipose depots ex vivo. I: Fold glycerol increase in the media of isoproterenol-stimulated lipolysis in Adipo-VD white and brown differentiated preadipocytes from their respective depots. ${ }^{*} P<0.05$ versus - rtTA.

\section{Adipo-VD Mice Fatty Acid Handling Is Largely Unchanged}

Elevated lipids and systemic lipotoxicity have been identified as the primary driver of insulin resistance in obesity. Lymphatic vessels are a route of both dietary fatty acid transport as well as peripheral reverse cholesterol transport. ${ }^{25}$ Differences in lipid handling in Adipo-VD mice were, therefore, determined. Fasting triglyceride levels were unchanged in Adipo-VD mice regardless of diet (Figure 3A), although circulating cholesterol levels were surprisingly lower in obese Adipo-VD mice (Figure 3B). Oral triglyceride delivery and clearance were unchanged in obese Adipo-VD mice compared with littermates (Figure 3C); a small early increase in appearance was measured under chow-fed conditions (Supplemental Figure S5A). Despite this lack of circulating differences, obese Adipo-VD livers contained significantly less triglyceride (Figure 3D), suggesting Adipo-VD adipose tissue was efficiently storing lipid.

Quantitative PCR analysis of s.c. adipose tissue demonstrated a variably increased expression of adipocyte browning genes, with Prdml6 being significantly elevated (Figure 3E). Mirroring these findings, protein levels of uncoupling protein 1 were elevated in obese Adipo-VD IWAT (Figure 3F) by immunoblot; increased uncoupling protein 1 protein was also measured in chow-fed Adipo-VD white adipose (Supplemental Figure S5B). To directly measure lipid uptake and oxidation in tissues, ${ }^{3} \mathrm{H}$-triolein, a triglyceride analog, was infused intravenously. ${ }^{3} \mathrm{H}$ counts in 
A
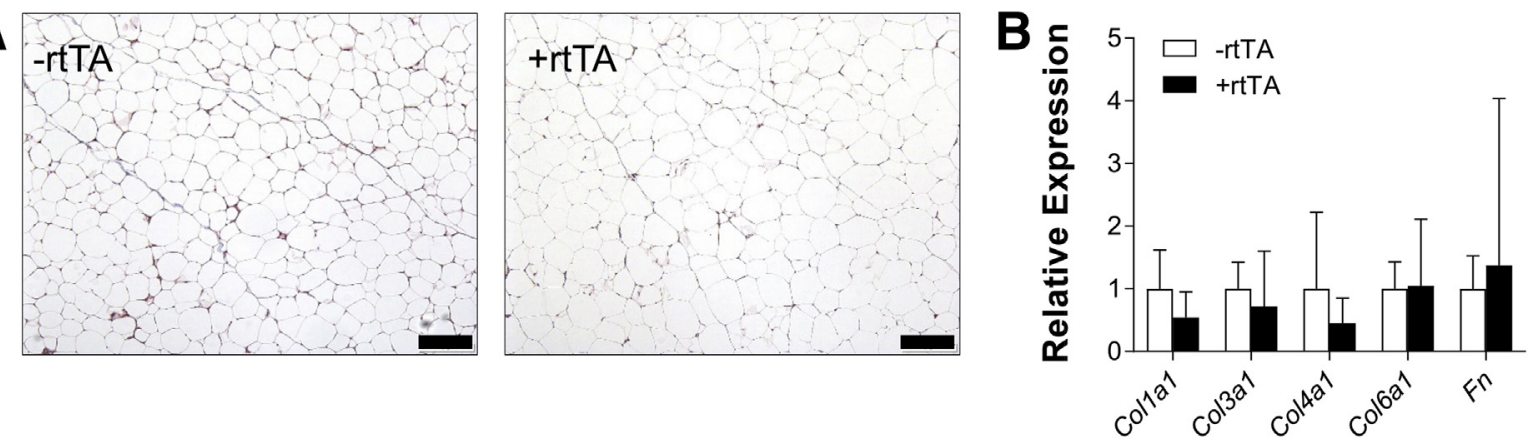

C

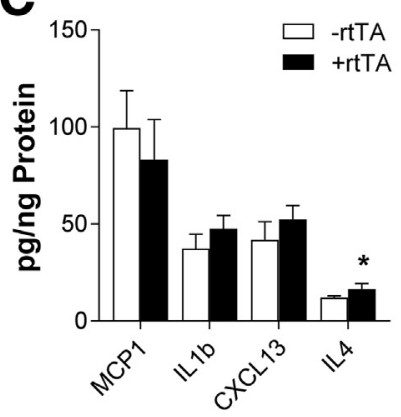

E

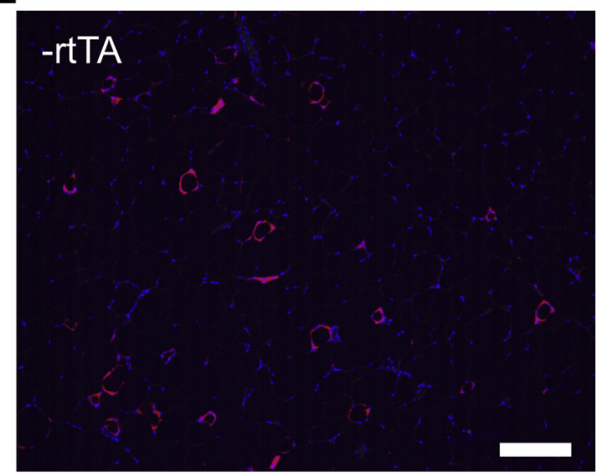

D
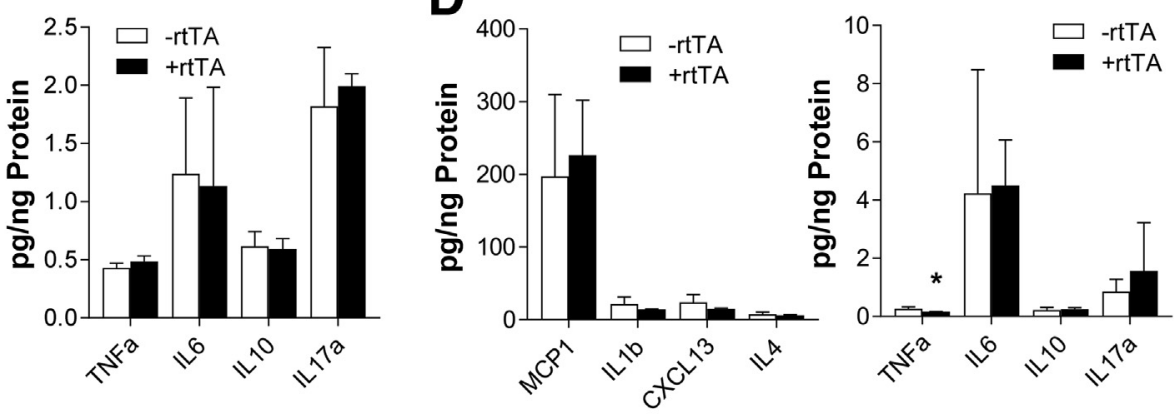

$\mathbf{F}$
G

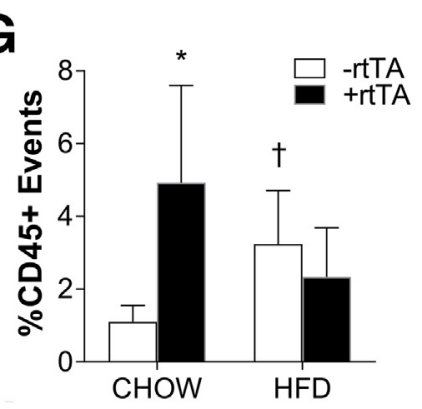

\section{$+r t$ TA}
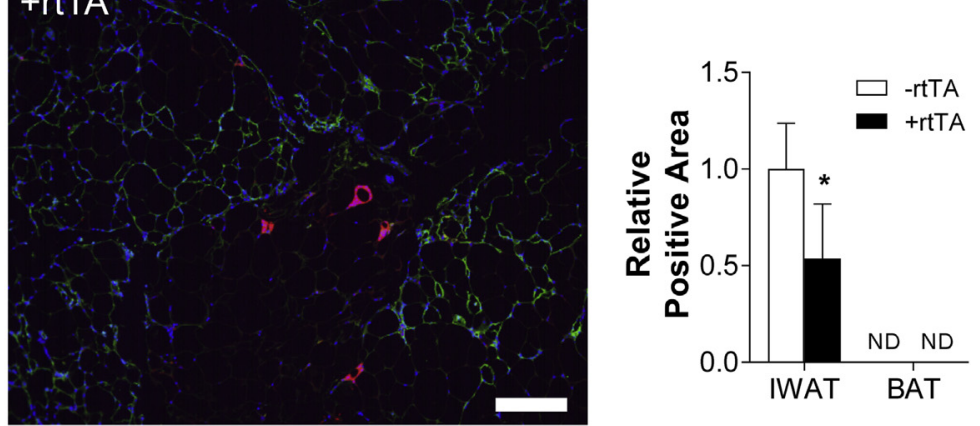

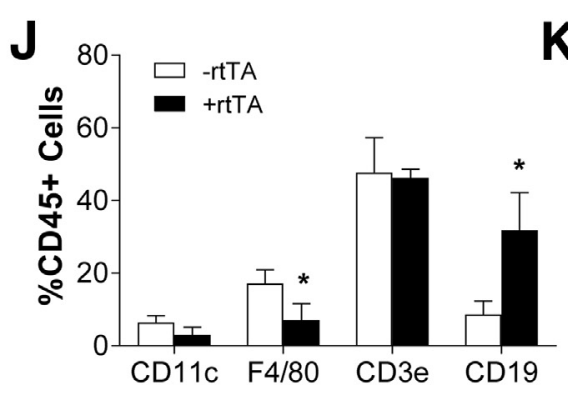

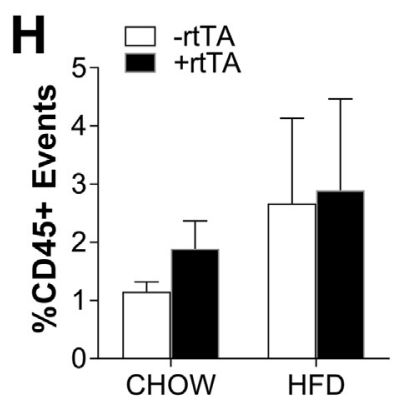

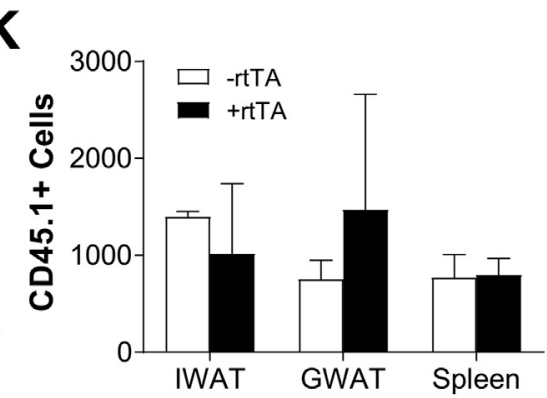

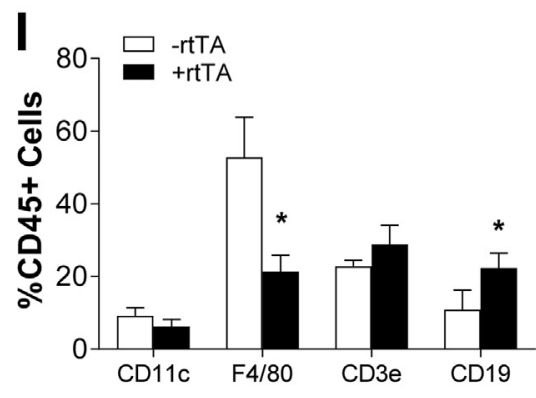

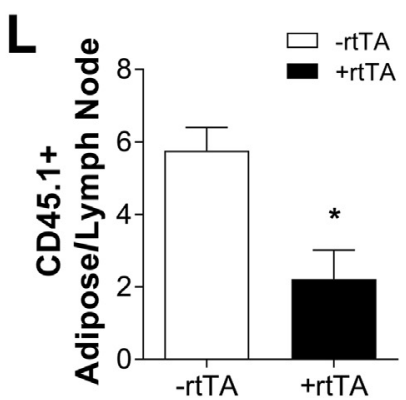


the lipid and aqueous fractions of tissues represent the uptake and oxidation of tissues, respectively. Across the eight tissues measured, only gonadal adipose tissue demonstrated significantly increased triglyceride uptake in obese AdipoVD mice (Figure 3G). Oxidation was largely unchanged across the tissues of obese mice (Figure $3 \mathrm{H}$ ); chow-fed mice also exhibited no remarkable differences (Supplemental Figure S5, C and D). Thus, overall basal lipid handling was similar. Adipo-VD mice on a chow diet demonstrated reduced dark cycle respiratory exchange ratio, food intake, and movement in metabolic cage studies (Supplemental Figure S5, E-H). Obese Adipo-VD mice, however, exhibited an increased cumulative respiratory exchange ratio and ate more during dark cycles than their littermates (Figure 3, I-K, and Supplemental Figure S5I). No differences were measured in heat production in either lean or obese Adipo-VD mice (Supplemental Figure S5, J and K), and leptin levels were comparable in obese Adipo-VD mice (Supplemental Figure S5L). Obese Adipo-VD mouse white adipose tissue thus appeared to function with only minor differences in lipid uptake and storage.

\section{Increased Adipose Lymphatic Density Enhances Glycerol Flux during Lipolysis}

Glycerol is one of the few metabolites found to be enriched in peripheral lymph over venous blood. ${ }^{26}$ It was, therefore, studied whether increased adipose lymphatic density improved glycerol flux from adipose tissue during lipolysis.

After $\beta-3$ adrenergic receptor stimulation, chow-fed Adipo-VD mice demonstrated a rapid and significant increase in circulating glycerol levels (Figure 4A). Increased circulating free fatty acid levels, however, were equivalent to Adipo-VD littermates, suggesting no overall enhancement of lipolysis per se in Adipo-VD adipose (Figure 4B). Serum glucose levels decreased equally after adrenergic stimulation (Figure 4C). Obese Adipo-VD mice also demonstrated significantly higher serum glycerol levels during lipolysis (Figure 4D) with, like under chow-fed conditions, equivalent free fatty acid levels (Figure 4E). Further demonstrating the enhanced glucose disposal in obese Adipo-VD mice compared with their littermates, serum glucose levels decreased significantly during lipolysis: a remarkable response not normally measured in obese, insulin-resistant mice (Figure 4F). In addition, obese AdipoVD inguinal adipose tissues demonstrated reduced hormone-sensitive lipase phosphorylation after insulin injection (Figure 4G), suggesting improved lipid storage.

To test that changes in glycerol flux were not inherent to the Adipo-VD adipocyte endogenous lipolytic capacity, IWAT and gonadal visceral white adipose depots were removed from Adipo-VD and littermate mice and treated with isoproterenol, a $\beta-1$ adrenergic receptor stimulant, ex vivo. No differences were measured in media glycerol levels after 1-hour stimulation (Figure 4H). Similarly, preadipocytes derived from Adipo-VD and littermate inguinal and brown adipose depots and differentiated in the presence of doxycycline displayed no difference in basal or isoproterenol-stimulated lipolysis (Figure 4I). Elevated serum glycerol levels during lipolysis thus appear to be through lymphatic transport or some factor only present in vivo.

\section{VEGF-D Expression Does Not Increase Fibrosis or Inflammation in Obese Adipo-VD Adipose}

Fibrosis, inflammation, and immune accumulation are indicative of dysfunctional adipose in obesity. Absent other inflammatory stimuli, increased VEGFR-3 signaling can recruit tissue macrophages. ${ }^{15,27}$ Under high-fat diet challenge, Adipo-VD mice displayed no increase in fibrosis in s.c. inguinal adipose tissue judged by trichrome staining (Figure 5A), and genes for common adipose tissue collagens were not increased compared with obese littermates (Figure 5B). Elevated VEGF-D levels in Adipo-VD mice are, therefore, not more profibrotic under obese conditions.

Similar to the fibrotic response, inflammatory cytokine protein levels, measured by Luminex Multiplex assay (MilliporeSigma, Burlington, MA), were not increased in obese Adipo-VD mouse IWAT (Figure 5C). Only IL-4, a type 2 helper T-cell cytokine, was significantly elevated. Gonadal adipose tissue protein profiling demonstrated a similar response, with no changes measured (Supplemental Figure S6A). Brown adipose tissue, which had higher VEGF-D induction than white depots in obese Adipo-VD mice, likewise exhibited no increase in inflammatory proteins measured; tumor necrosis factor- $\alpha$ levels were reduced (Figure 5D). Obese Adipo-VD mice do not display

\footnotetext{
Figure 5 Inflammation and immune accumulation in obese Adipo-VD mice. A: Trichrome staining of obese wild-type and Adipo-VD mouse inguinal white adipose tissue (IWAT) after 4 months of high-fat diet (HFD) feeding. B: Expression of adipose matrix RNA in obese inguinal adipose tissue. C: Cytokine protein levels in Adipo-VD inguinal white adipose tissue protein homogenate quantified by multiplex array. Graphs are split because of axis range. D: Cytokine protein levels in Adipo-VD brown adipose tissue (BAT) protein homogenate quantified by multiplex array. Graphs are split because of axis range. E: Macrophage crownlike structures (red; Mac-2) in Adipo-VD inguinal adipose tissue. Locations of Mac-2 labeling are random and not associated with lymphatic structures (green; LYVE-1). F: Quantitation of immunofluorescence images of Mac-2 labeling in Adipo-VD IWAT and BAT. Labeling was not detected (ND) in any BAT images. G: Percentage of counted events that represent immune cells $\left(\mathrm{CD}_{4} 5^{+}\right)$in chow- and high-fat diet-fed inguinal adipose. H: Percentage of counted events that represent immune cells $\left(C D 45^{+}\right)$in chow- and high-fat diet-fed gonadal adipose. I: Dendritic cell (CD11c), macrophage (F4/80), T-cell (CD3e), and B-cell (CD19) populations in chow-fed inguinal adipose tissue of Adipo-VD mice. J: The same populations identified in obese inguinal adipose tissue. K: Total numbers of CD45.1 $1^{+}$cells identified in obese IWAT and gonadal white adipose tissue (GWAT) depots and the spleens of Adipo-VD mice 24 hours after infusion. L: Ratio of CD45.1 ${ }^{+}$immune cells remaining in the adipose tissue/those having trafficked to the inguinal lymph node in obese Adipo-VD mice. ${ }^{*} P<0.05$ versus $-\mathrm{rtTA}^{\dagger}{ }^{\dagger} P<0.05$ versus chow. Scale bars: $200 \mu \mathrm{m}$ (A); $100 \mu \mathrm{m}$ (E). ND, not determined.
} 
increased adipose inflammation and suggest a potential reduction.

Resident immune cells were reduced in obese Adipo-VD mouse adipose. Fewer Mac- $2^{+}$macrophage crown-like structures were identified and quantified in Adipo-VD inguinal adipose tissue (Figure 5, E and F). No Mac- $2^{+}$ cells were identified within brown adipose tissue (Figure 5F). Flow cytometry analysis of chow and obese Adipo-VD white adipose depots was performed. Under chow-fed conditions, VEGF-D increased the number of CD $45^{+}$immune cells in inguinal and gonadal adipose tissue (Figure 5, G and H). F4/80 ${ }^{+}$macrophages were identified as the predominant immune population in both depots (Figure 5I and Supplemental Figure S6B). In Adipo-VD inguinal adipose, however, the proportion of $\mathrm{F} 4 / 80^{+}$cells was decreased, and $\mathrm{CD}_{1}{ }^{+} \mathrm{B}$ cells were increased (Figure 5I). This shift was not seen in the gonadal adipose (Supplemental Figure S6B). During obesity, increased numbers of immune cells are present in adipose tissues (Figure 5G), with increased $\mathrm{CD} 3 \mathrm{e}^{+}$T-cell infiltration (Figure 5J and Supplemental Figure S6C). In obese Adipo-VD inguinal adipose, however, there was no increase in total $\mathrm{CD}^{4} 5^{+}$cells (Figure $5 \mathrm{G}$ ). Similar to chow-fed conditions, adipose VEGF-D expression shifted the relative populations of immune cell types, with a reduced proportion of $\mathrm{F} 4 / 80^{+}$macrophages (confirming the crown-like structure quantitation) and increased $\mathrm{CD}^{+} 9^{+} \mathrm{B}$ cells (Figure 5J). In gonadal adipose, which exhibited limited VEGF-D expression or lymphangiogenesis, no changes in immune populations were identified (Supplemental Figure S6C). Splenic populations were unchanged on chow (Supplemental Figure 6D). A small decrease in CD19+ cells was found in the spleens of obese Adipo-VD mice (Supplemental Figure 6E).

To determine if increased lymphatics provide a route for immune cell efflux from Adipo-VD adipose tissue, splenocytes were isolated from FVB mice that carry the CD45.1 isoform and infused into obese Adipo-VD mice and littermates (that carry the CD45.2 isoform). The numbers of CD $45.1^{+}$cells identified in IWAT and gonadal visceral white adipose depots were the same (Figure $5 \mathrm{~K}$ ), indicating that trafficking into the tissues was largely unchanged. The ratio of CD45.1 $1^{+}$cells within the inguinal depot compared with the inguinal lymph node-the presumed drainage point of adipose lymphatics-was, however, significantly reduced (Figure 5L), suggesting increased trafficking via lymphatics from the adipose interstitium.

Adipo-VD mice challenged with a standard commercial $60 \% \mathrm{kcal}$ from fat diet (a mix of fatty acids) were metabolically healthier than their littermates. If Adipo-VD mice were made obese on a diet containing mostly unsaturated fatty acids, they also performed better during an oral glucose challenge (Figure 6, A and B). Saturated fatty acids, particularly palmitate, increase inflammation further. ${ }^{28}$ Adipo-VD mice fed a high-fat diet composed of mostly palmitate did not demonstrate improved glucose handling
(Figure 6, C and D). Obese Adipo-VD mice are metabolically improved, in part through a limited reduction in adipose tissue inflammation or immune accumulation.

\section{Discussion}

The roles of inflammation-induced lymphangiogenesis in fluid, macromolecule, and immune cell clearance make the lymphatic vasculature an attractive potential target in adipose tissue inflammation. ${ }^{7,29}$ Obesity, however, alters lymphatic architecture and reduces lymphatic vessel function, and dysfunctional lymphatic vessels result in local adipose tissue expansion-a vicious cycle highlighting the potential importance of lymphatic circulation in metabolic

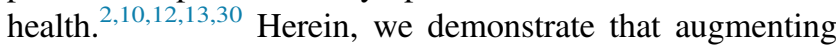
VEGF-D signaling specifically in adipose tissue induces lymphangiogenesis and improves glucose and lipid homeostasis during obesity. Multiple factors appear to contribute to this improvement. Systemic glucose handling was improved and liver lipid deposition was reduced in obese Adipo-VD mice. Adipo-VD mice demonstrated improved respiration and well-being despite similar weight and adiposity. Fewer adipose resident macrophages were found in Adipo-VD white adipose tissues. Although each effect was individually small, in total, chronically elevated VEGF-D signaling and an increased presence of lymphatic structures in Adipo-VD mouse adipose tissue reduced adipose tissue dysfunction in obesity.

Inflammatory adipose tissue expansion in obesity has been identified as the primary driver of the metabolic syndrome etiology. Rapid adipose expansion is characterized by hypoxia, immune cell residency, and tissue fibrosis, resulting in adipocytes that fail to properly store excess calories. ${ }^{1,31}$ Dysregulated lipolysis in adipocytes leads to increased circulating lipid levels and ectopic lipid deposition in other tissues, resulting in their own insulin resistance. In preclinical studies that have increased obese adipose tissue blood vascularization, eliminated immune cell populations, or manipulated extracellular matrix fibrosis, adipose tissue health and systemic glucose homeostasis are restored. ${ }^{32,33}$ VEGFR-3 signaling has been previously linked to increase inflammation and reduce insulin sensitivity in obesity. ${ }^{22,27}$ Mice expressing elevated VEGF-C under the keratin 14 promoter demonstrate metabolic dysfunction on chow diet that continues when mice are challenged with high-fat feeding. ${ }^{27}$ Elevated numbers of CD11b macrophages were identified in mice expressing elevated VEGF-C under the keratin 14 promoter s.c. IWAT. ${ }^{27}$ In a similar study, systemic blockade of VEGFR-3 signaling during obesity reduced the chemotactic effects of endogenous VEGFR-3 ligands in adipose tissues, which are elevated in obesity, and thereby reduced adipose macrophage numbers and improved metabolism. ${ }^{22}$ These findings are in complete agreement with the current study. Indeed, we demonstrate that in our chow-fed Adipo-VD mice with adipose tissue otherwise at a healthy homeostasis, specifically elevating VEGF-D levels 

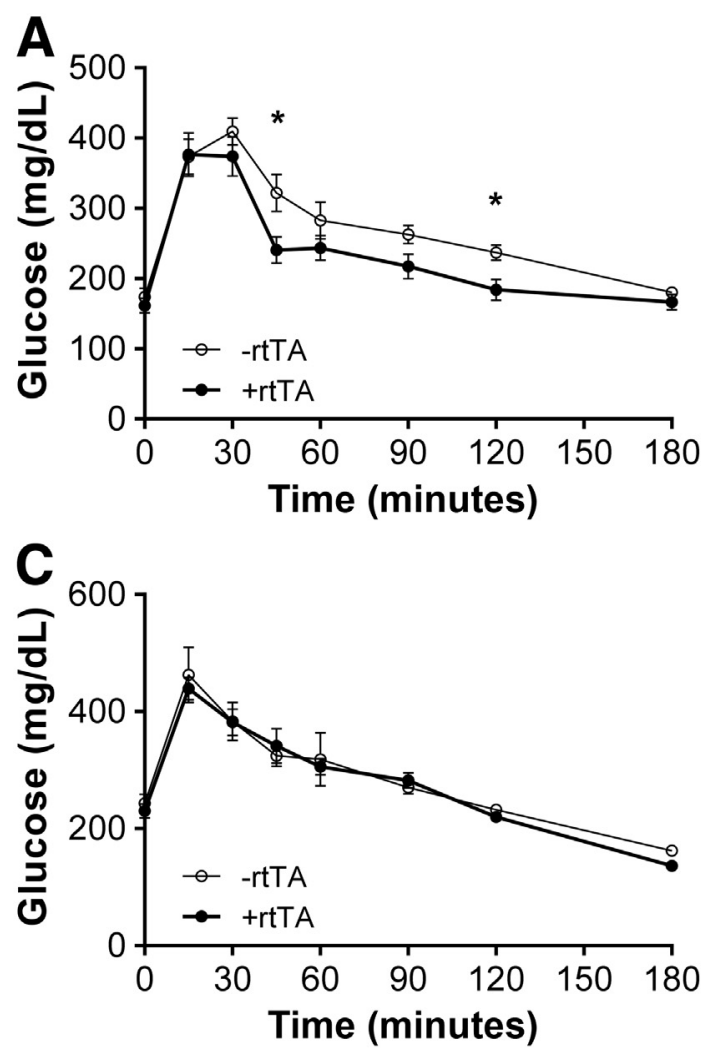

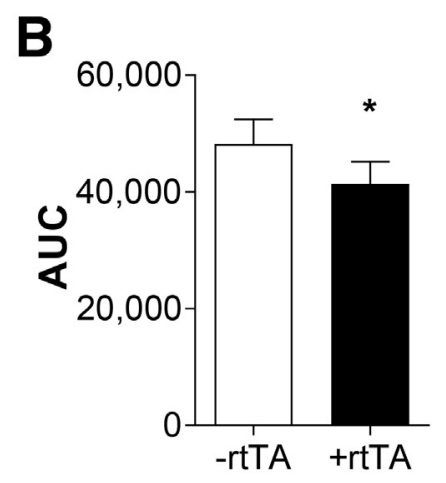

D

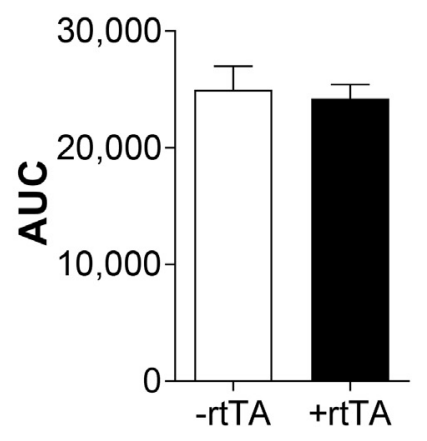

Figure 6 Ability of VEGF-D induction to overcome inflammation. A: Glucose levels during oral glucose tolerance test of doxycycline-high-unsaturated fat diet-fed Adipo-VD mice at 4 months. B: Average area under the curve (AUC) calculated from glucose levels in previous test. C: Glucose levels during oral glucose tolerance test of doxycycline high-saturated fat diet-fed Adipo-VD mice at 4 months. D: Average area under the curve calculated from glucose levels in previous test. $n=5-\operatorname{rtTA}(\mathbf{A}$ and $\mathbf{B}) ; n=6+\mathrm{rtTA}(\mathbf{A}$ and $\mathbf{B})$; $n=4-\mathrm{rtTA}$ (C and D); $n=5+\mathrm{rtTA}$ (C and $\mathbf{D})$. ${ }^{*} P<0.05$ versus - rtTA. is not only lymphangiogenic, but is also chemotactic, inducing fibrosis, macrophage accumulation, and elevated cytokine levels in adipose tissue. ${ }^{15}$ The current obesity study is different from past work, however, because mice expressing elevated VEGF-C under the keratin 14 promoter or systemic VEGFR-3 blockade did not alter adipose lymphangiogenesis; Adipo-VD mice are the first demonstration of this potential. In obesity, when the overall endogenous inflammatory milieu is increased, the current data demonstrate that a modest increase in VEGF-D levels and the novel induction of adipose lymphangiogenesis achieved in Adipo-VD mice appear to supersede this inflammatory effect with a now equivalent, or better, cytokine profile and reduced total immune cell numbers. Adipo-VD mice demonstrate little to no change in visceral adipose lymphatics, suggesting that the IWAT and BAT depots depend on progenitors from the skin (a unique, nonvenous pool $^{34}$ ) such that the lymphangiogenic gradient must initiate from the adipose and not be inhibited/induced from skinspecific transgene expression. Identifying the origin of these new lymphatics or other potential roles of adipose-specific VEGFR-3 signaling in obesity are thus unique to the AdipoVD mouse.

Properly functioning lymphatic vessels remove fluid, antigens, and cytokines and provide for immune cell egress from peripheral tissues. This reduces the local inflammatory burden and allows for a regulated immune response in the draining lymph node., 5,35 Recent research has also highlighted an emerging role for LECs to function as antigen-presenting cells, induce dendritic cell activation, and regulate T-lymphocyte function. ${ }^{6,36,37}$ IAL has the potential to form tertiary lymphoid organs: peripheral sites of lymphatic-immune interaction and regulation. ${ }^{37-39}$ AdipoVD exhibited reduced total immune cell numbers and increased trafficking from adipose tissue to the sentinel lymph node, resulting in improved tissue health; whether this is by immune cell removal or aiding in the immune regulation of tissue remodeling remains to be identified in our model. VEGF-D and/or lymphatics appear to increase adipose B-cell numbers in Adipo-VD mice, suggesting a potential change in the adaptive immune response or tertiary lymphoid organ formation. Further immune/inflammatory profiling may identify these changes. It will be exciting to identify these mechanisms because they are potentially relevant to other adipose-associated conditions, such as lymphedematous adipose tissue, pathologic lipedema, and Crohn disease. ${ }^{7}$

Lymphatic vessel fluid and macromolecule transport is also important for adipose homeostasis. Leaky collecting lymphatic vessels can drive adipogenesis, ${ }^{40,41}$ and severed lymphatics, as in lymphedema, result in upstream adipose expansion. ${ }^{10,42}$ Genetically correcting leaky lymphatics prevents obesity in mice. ${ }^{43}$ Although we were unable to determine how expanded adipose lymphatics alter tissue hydrostatic and osmotic gradients, the Adipo-VD mouse may have revealed a previously unappreciated role for lymphatic metabolite flux. Human studies have identified higher 
interstitial glycerol concentrations in obese adipose than venous blood, and glycerol is the sole metabolite enriched in lymph. $^{26,44,45}$ It was identified that increased lymphatic density may provide a route for increased glycerol transport from the adipose tissue interstitium during $\beta-3$ adrenergic stimulated lipolysis in Adipo-VD mice. Whether VEGF-D signaling regulates other soluble factors or the physiology of other metabolic regulatory systems (eg, adipose-central feedback or in cholesterol homeostasis ${ }^{46}$ ) remains to be fully elucidated and would make other potentially VEGFR-3 expressing cells new targets in disease.

Lymphatic vessels and adipose tissue share an intimate relationship in both their proximity and roles in lipid handling. ${ }^{2,11}$ Although the overall metabolic effect in obese Adipo-VD mice is subtle, the mouse model now provides a novel platform on which to further identify lymphatic capillary roles in adipose tissue health and metabolism. An increased appreciation of these roles should make targeting lymphatic endothelial cell biology and lymphatic vessel function part of any integrated approach toward obesity and other diseases of adipose inflammation.

\section{Acknowledgments}

We thank Selene Howe (Texas A\&M College of Medicine and Texas A\&M Institute for Genome Sciences and Society) and David Threadgill (Texas A\&M College of Medicine and Texas A\&M Institute for Genome Sciences and Society) for assistance with and use of the metabolic cages, and Michael Dellinger (University of Texas Southwestern Medical Center at Dallas) for performing and capturing the LYVE-1 intestinal imaging.

\section{Supplemental Data}

Supplemental material for this article can be found at https://doi.org/10.1016/j.ajpath.2018.12.008.

\section{References}

1. Rutkowski JM, Stern JH, Scherer PE: The cell biology of fat expansion. J Cell Biol 2015, 208:501-512

2. Rutkowski JM, Davis KE, Scherer PE: Mechanisms of obesity and related pathologies: the macro- and microcirculation of adipose tissue. FEBS J 2009, 276:5738-5746

3. Sun K, Kusminski CM, Scherer PE: Adipose tissue remodeling and obesity. J Clin Invest 2011, 121:2094-2101

4. Sun K, Tordjman J, Clement K, Scherer PE: Fibrosis and adipose tissue dysfunction. Cell Metab 2013, 18:470-477

5. Wiig H, Swartz MA: Interstitial fluid and lymph formation and transport: physiological regulation and roles in inflammation and cancer. Physiol Rev 2012, 92:1005-1060

6. Card CM, Yu SS, Swartz MA: Emerging roles of lymphatic endothelium in regulating adaptive immunity. J Clin Invest 2014, 124: 943-952

7. Abouelkheir GR, Upchurch BD, Rutkowski JM: Lymphangiogenesis: fuel, smoke, or extinguisher of inflammation's fire? Exp Biol Med (Maywood) 2017, 242:884-895
8. Gousopoulos E, Karaman S, Proulx ST, Leu K, Buschle D, Detmar M: High-fat diet in the absence of obesity does not aggravate surgically induced lymphoedema in mice. Eur Surg Res 2017, 58:180-192

9. Lee Y, Fluckey JD, Chakraborty S, Muthuchamy M: Hyperglycemiaand hyperinsulinemia-induced insulin resistance causes alterations in cellular bioenergetics and activation of inflammatory signaling in lymphatic muscle. FASEB J 2017, 31:2744-2759

10. Mehrara BJ, Greene AK: Lymphedema and obesity: is there a link? Plast Reconstr Surg 2014, 134:154e-160e

11. Harvey NL: The link between lymphatic function and adipose biology. Ann N Y Acad Sci 2008, 1131:82-88

12. Arngrim N, Simonsen L, Holst JJ, Bulow J: Reduced adipose tissue lymphatic drainage of macromolecules in obese subjects: a possible link between obesity and local tissue inflammation? Int J Obes (Lond) 2013, 37:748-750

13. Nitti MD, Hespe GE, Kataru RP, Garcia Nores GD, Savetsky IL, Torrisi JS, Gardenier JC, Dannenberg AJ, Mehrara BJ: Obesity-induced lymphatic dysfunction is reversible with weight loss. J Physiol 2016, 594:7073-7087

14. Aspelund A, Robciuc MR, Karaman S, Makinen T, Alitalo K: Lymphatic system in cardiovascular medicine. Circ Res 2016, 118 $515-530$

15. Lammoglia GM, Van Zandt CE, Galvan DX, Orozco JL, Dellinger MT, Rutkowski JM: Hyperplasia, de novo lymphangiogenesis, and lymphatic regression in mice with tissue-specific, inducible overexpression of murine VEGF-D. Am J Physiol Heart Circ Physiol 2016, 311:H384-H394

16. Baldwin ME, Catimel B, Nice EC, Roufail S, Hall NE, Stenvers KL, Karkkainen MJ, Alitalo K, Stacker SA, Achen MG: The specificity of receptor binding by vascular endothelial growth factor-d is different in mouse and man. J Biol Chem 2001, 276: 19166-19171

17. Sun K, Wernstedt Asterholm I, Kusminski CM, Bueno AC, Wang ZV, Pollard JW, Brekken RA, Scherer PE: Dichotomous effects of VEGFA on adipose tissue dysfunction. Proc Natl Acad Sci U S A 2012, 109: $5874-5879$

18. Wang ZV, Deng Y, Wang QA, Sun K, Scherer PE: Identification and characterization of a promoter cassette conferring adipocyte-specific gene expression. Endocrinology 2010, 151:2933-2939

19. Xia JY, Holland WL, Kusminski CM, Sun K, Sharma AX, Pearson MJ, Sifuentes AJ, McDonald JG, Gordillo R, Scherer PE: Targeted induction of ceramide degradation leads to improved systemic metabolism and reduced hepatic steatosis. Cell Metab 2015, 22: 266-278

20. Asterholm IW, Rutkowski JM, Fujikawa T, Cho YR, Fukuda M, Tao C, Wang ZV, Gupta RK, Elmquist JK, Scherer PE: Elevated resistin levels induce central leptin resistance and increased atherosclerotic progression in mice. Diabetologia 2014, 57: $1209-1218$

21. Singh S, Rajput YS, Barui AK, Sharma R, Datta TK: Fat accumulation in differentiated brown adipocytes is linked with expression of Hox genes. Gene Expr Patterns 2016, 20:99-105

22. Karaman S, Hollmen M, Robciuc MR, Alitalo A, Nurmi H, Morf B, Buschle D, Alkan HF, Ochsenbein AM, Alitalo K, Wolfrum C, Detmar M: Blockade of VEGF-C and VEGF-D modulates adipose tissue inflammation and improves metabolic parameters under high-fat diet. Mol Metab 2015, 4:93-105

23. Nitschke M, Bell A, Karaman S, Amouzgar M, Rutkowski JM, Scherer PE, Alitalo K, McDonald DM: Retrograde lymph flow leads to chylothorax in transgenic mice with lymphatic malformations. Am J Pathol 2017, 187:1984-1997

24. Cho CH, Koh YJ, Han J, Sung HK, Jong Lee H, Morisada T, Schwendener RA, Brekken RA, Kang G, Oike Y, Choi TS, Suda T, Yoo OJ, Koh GY: Angiogenic role of LYVE-1-positive macrophages in adipose tissue. Circ Res 2007, 100:e47-e57

25. Randolph GJ, Miller NE: Lymphatic transport of high-density lipoproteins and chylomicrons. J Clin Invest 2014, 124:929-935 
26. Miller NE, Michel CC, Nanjee MN, Olszewski WL, Miller IP, Hazell M, Olivecrona G, Sutton P, Humphreys SM, Frayn KN: Secretion of adipokines by human adipose tissue in vivo: partitioning between capillary and lymphatic transport. Am J Physiol Endocrinol Metab 2011, 301:E659-E667

27. Karaman S, Hollmen M, Yoon SY, Alkan HF, Alitalo K, Wolfrum C, Detmar M: Transgenic overexpression of VEGF-C induces weight gain and insulin resistance in mice. Sci Rep 2016, 6:31566

28. Shi H, Kokoeva MV, Inouye K, Tzameli I, Yin H, Flier JS: TLR4 links innate immunity and fatty acid-induced insulin resistance. J Clin Invest 2006, 116:3015-3025

29. Escobedo N, Oliver G: The lymphatic vasculature: its role in adipose metabolism and obesity. Cell Metab 2017, 26:598-609

30. Blum KS, Karaman S, Proulx ST, Ochsenbein AM, Luciani P, Leroux JC, Wolfrum C, Detmar M: Chronic high-fat diet impairs collecting lymphatic vessel function in mice. PLoS One 2014, 9: e94713

31. Stern JH, Rutkowski JM, Scherer PE: Adiponectin, leptin, and fatty acids in the maintenance of metabolic homeostasis through adipose tissue crosstalk. Cell Metab 2016, 23:770-784

32. Crewe C, An YA, Scherer PE: The ominous triad of adipose tissue dysfunction: inflammation, fibrosis, and impaired angiogenesis. J Clin Invest 2017, 127:74-82

33. Kusminski CM, Bickel PE, Scherer PE: Targeting adipose tissue in the treatment of obesity-associated diabetes. Nat Rev Drug Discov 2016, 15:639-660

34. Martinez-Corral I, Ulvmar MH, Stanczuk L, Tatin F, Kizhatil K, John SW, Alitalo K, Ortega S, Makinen T: Nonvenous origin of dermal lymphatic vasculature. Circ Res 2015, 116:1649-1654

35. Kim H, Kataru RP, Koh GY: Inflammation-associated lymphangiogenesis: a double-edged sword? J Clin Invest 2014, 124:936-942

36. Hirosue S, Vokali E, Raghavan VR, Rincon-Restrepo M, Lund AW, Corthesy-Henrioud P, Capotosti F, Halin Winter C, Hugues S, Swartz MA: Steady-state antigen scavenging, cross-presentation, and CD8 + T cell priming: a new role for lymphatic endothelial cells. J Immunol 2014, 192:5002-5011
37. Maisel K, Sasso MS, Potin L, Swartz MA: Exploiting lymphatic vessels for immunomodulation: rationale, opportunities, and challenges. Adv Drug Deliv Rev 2017, 114:43-59

38. Swartz MA: Immunomodulatory roles of lymphatic vessels in cancer progression. Cancer Immunol Res 2014, 2:701-707

39. Ruddle NH: Lymphatic vessels and tertiary lymphoid organs. J Clin Invest 2014, 124:953-959

40. Harvey NL, Srinivasan RS, Dillard ME, Johnson NC, Witte MH, Boyd K, Sleeman MW, Oliver G: Lymphatic vascular defects promoted by Prox 1 haploinsufficiency cause adult-onset obesity. Nat Genet 2005, 37:1072-1081

41. Kuan EL, Ivanov S, Bridenbaugh EA, Victora G, Wang W, Childs EW, Platt AM, Jakubzick CV, Mason RJ, Gashev AA, Nussenzweig M, Swartz MA, Dustin ML, Zawieja DC, Randolph GJ: Collecting lymphatic vessel permeability facilitates adipose tissue inflammation and distribution of antigen to lymph node-homing adipose tissue dendritic cells. J Immunol 2015, 194:5200-5210

42. Rutkowski JM, Moya M, Johannes J, Goldman J, Swartz MA: Secondary lymphedema in the mouse tail: lymphatic hyperplasia, VEGF-C upregulation, and the protective role of MMP-9. Microvasc Res 2006, 72:161-171

43. Escobedo N, Proulx ST, Karaman S, Dillard ME, Johnson N, Detmar M, Oliver G: Restoration of lymphatic function rescues obesity in Prox1-haploinsufficient mice. JCI Insight 2016, 1:e85096

44. Jansson PA, Smith U, Lonnroth P: Interstitial glycerol concentration measured by microdialysis in two subcutaneous regions in humans. Am J Physiol 1990, 258(Pt 1):E918-E922

45. Jocken JW, Goossens GH, Boon H, Mason RR, Essers Y, Havekes B, Watt MJ, van Loon LJ, Blaak EE: Insulin-mediated suppression of lipolysis in adipose tissue and skeletal muscle of obese type 2 diabetic men and men with normal glucose tolerance. Diabetologia 2013, 56: $2255-2265$

46. Tirronen A, Vuorio T, Kettunen S, Hokkanen K, Ramms B, Niskanen H, Laakso H, Kaikkonen MU, Jauhiainen M, Gordts P, Yla-Herttuala S: Deletion of lymphangiogenic and angiogenic growth factor VEGF-D leads to severe hyperlipidemia and delayed clearance of chylomicron remnants. Arterioscler Thromb Vasc Biol 2018, 38:2327-2337 